\title{
Le gisement acheuléen de Cagny-l'Épinette (Somme)
}

Alain Tuffreau, Pierre Antoine, Philip G. Chase, Harold L. Dibble, Brooks B. Ellwood, Thijs van Kolfschoten, Agnès Lamotte, Michel Laurent, Shannon P. Mc Pherron, AnneMarie Moigne, André-Valentin Munaut

\section{Citer ce document / Cite this document :}

Tuffreau Alain, Antoine Pierre, Chase Philip G., Dibble Harold L., Ellwood Brooks B., van Kolfschoten Thijs, Lamotte Agnès, Laurent Michel, Mc Pherron Shannon P., Moigne Anne-Marie, Munaut André-Valentin. Le gisement acheuléen de Cagnyl'Épinette (Somme). In: Bulletin de la Société préhistorique française, tome 92, n², 1995. pp. 169-192;

doi : 10.3406/bspf.1995.10004

http://www.persee.fr/doc/bspf_0249-7638_1995_num_92_2_10004

Document généré le 24/02/2017 


\begin{abstract}
steppic landscape interrupted by trees. The climatic conditions were close to those of an interglacial, from the Tardiglacial to the Early Glacial. The lithic industries are characterized by bifaces with various morphologies. The flaking and blank production indicate an opportunistic behaviour which did not develop any sophisticated operating scheme. On the whole non-standardized flake tools were badly manufactured : they mainly consist of notches and denti- culates. The big fauna is typical of the Middle Pleistocene one (Bos primigenius trochoceros, Equus caballus mosbachensis, Equus hydruntinus, Cervus elaphus, Dama...). Comparisons with other site fauna could be drawn. When the spatial distribution analysis is fully achieved there will be a better understanding of bones and flint artefacts.
\end{abstract}

\title{
Résumé
}

RESUMÉ Le gisement acheuléen de Cagny- $\Gamma$ Épi nette a une séquence archéologique incluse dans les formations flu- vi at i les fines de la nappe la plus récente du complexe de la "moyenne terrasse" du bassin de la Somme et à la partie inférieure de la couverture sablo-limoneuse sus-jacente. D'après les données palynologiques et pa- léontologiques, les occupations humaines, attestées par la présence de silex taillés et d'ossements d'animaux montrant des signes d'actions an- thropiques, se situent dans un paysage de steppes arborées correspondant à des ambiances climatiques proches de l'interglaciaire, allant du Tardiglaciaire au Début Glaciaire. Les industries lithiques se caractérisent par la présence de bifaces de morphologie variée. Le débitage et la production des supports témoignent d'un comportement opportuniste sans chaîne opératoire élaborée. L'outillage sur éclat, non standardisé, comprend surtout des pièces de mauvaise facture, essentiellement des encoches et des denticulés. La grande faune correspond à des espèces caractéristiques du Pleistocene moyen (Bos primigenius trochoceros, Equus caballus mosbachensis, Equus hydruntinus, Cervus elaphus, Dama...). II a été possible d'établir des comparaisons avec les faunes d'autres gisements. Les études spatiales en cours permettront de mieux comprendre les relations entre les ossements et les silex taillés. 


\title{
LE GISEMENT ACHEULÉEN DE CAGNY-L'ÉPINETTE (SOMME)
}

\author{
Alain TUFFREAU, Pierre ANTOINE, Philip G. CHASE, Harold L. DIBBLE, Brooks B. ELLWOOD, \\ Thijs van KOLFSCHOTEN, Agnès LAMOTTE, Michel LAURENT, Shannon P. McPHERRON \\ Anne-Marie MOIGNE et André V. MUNAUT
}

\section{RÉSUME}

Le gisement acheuléen de Cagnyl'Épinette a une séquence archéologique incluse dans les formations fluviatiles fines de la nappe la plus récente du complexe de la "moyenne terrasse" du bassin de la Somme et à la partie inférieure de la couverture sablo-limoneuse sus-jacente. D'après les données palynologiques et paléontologiques, les occupations humaines, attestées par la présence de silex taillés et d'ossements d'animaux montrant des signes d'actions anthropiques, se situent dans un paysage de steppes arborées correspondant à des ambiances climatiques proches de l'interglaciaire, allant du Tardiglaciaire au Début Glaciaire.

Les industries lithiques se caractérisent par la présence de bifaces de morphologie variée. Le débitage et la production des supports témoignent d'un comportement opportuniste sans chaîne opératoire élaborée. L'outillage sur éclat, non standardisé, comprend surtout des pièces de mauvaise facture, essentiellement des encoches et des denticulés. La grande faune correspond à des espèces caractéristiques du Pléistocène moyen (Bos primigenius trochoceros, Equus caballus mosbachensis, Equus hydruntinus, Cervus elaphus, Dama...). II a été possible d'établir des comparaisons avec les faunes d'autres gisements. Les études spatiales en cours permettront de mieux comprendre les relations entre les ossements et les silex taillés.

\section{ABSTRACT}

Cagny-L'Épinette is an Acheulean site of the Somme basin. Its archaeological sequence is included in the fine fluvial formations of the most recent deposits of the "middle-terrace" complex and it is covered by a sandy and loamy deposit. The human occupations are witnessed by flaked and retouched flint artefacts as well as by animal bones showing anthropic activities. The pollen analysis and palaeontological studies revealed a steppic landscape interrupted by trees. The climatic conditions were close to those of an interglacial, from the Tardiglacial to the Early Glacial. The lithic industries are characterized by bifaces with various morphologies. The flaking and blank production indicate an opportunistic behaviour which did not develop any sophisticated operating scheme. On the whole non-standardized flake tools were badly manufactured : they mainly consist of notches and denticulates. The big fauna is typical of the Middle Pleistocene one (Bos primigenius trochoceros, Equus caballus mosbachensis, Equus hydruntinus, Cervus elaphus, Dama...). Comparisons with other site fauna could be drawn. When the spatial distribution analysis is fully achieved there will be a better understanding of bones and flint artefacts.

Le gisement de Cagny-l'Épinette est l'un des gisements acheuléens de la région d'Amiens (fig.1). II fait depuis 1980 l'objet de fouilles qui, pendant plusieurs années, ont surtout concerné la couverture sablo-li- moneuse et des sédiments fluviatiles fins peu épais comprenant des ossements (grands mammifères, micromammifères ; cf. : Tuffreau et al., 1986). Les cailloutis de la couverture contiennent des séries lithiques assez abondantes mais la faune n'est pas conservée dans ces niveaux. Les artefacts de la couverture sablo-limoneuse sont en position secondaire, ce qui accroît les risques de mélange entre des séries différentes et limite leur intérêt à leurs seules caractéristiques technologiques et typologiques. L'échantillonnage des séries de ces niveaux ayant été jugé suffisant, il fut décidé d'abandonner leur fouille, d'autant plus que la découverte en 1988 d'un chenal, où la séquence fluviatile fine se dilate nettement, nous amena à étudier de façon privilégiée cette partie du gisement. Dans l'Europe du Nord-Ouest, les dépôts fluviatiles fins sont parmi ceux qui sont le plus susceptibles de livrer des informations sur le contexte paléoclimatique en raison de la bonne conservation des ossements et des pollens. La dynamique fluviatile, assez faible, est en outre favorable à un enfouisse-

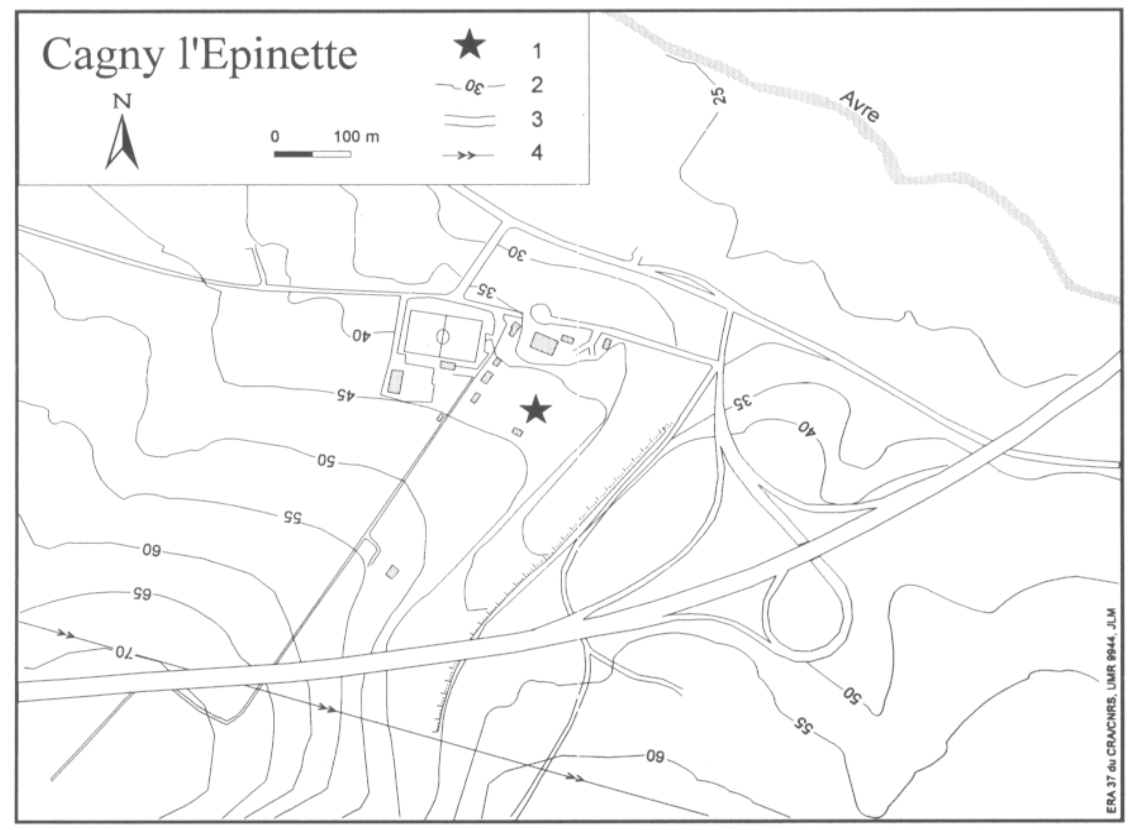

Fig. 1 - Cagny-|'Épinette. 1 : fouille archéologique ; 2 : courbe de niveau ; 3 : route ; 4 : ligne électrique moyenne tension. 
ment des vestiges archéologiques sans trop de déplacements. A partir de 1991, une équipe de l'Université de Pennsylvanie s'est jointe au programme d'étude du gisement de Cagny-l'Épinette. Cette collaboration permit notamment d'informatiser la fouille avec l'utilisation d'un théodolite électronique connecté avec un ordinateur portable.

La fouille du gisement de Cagnyl'Épinette n'est pas achevée et les différents travaux spécialisés sont à un degré d'avancement variable, ce qui rend difficile la présentation d'une synthèse équilibrée et d'une interprétation des niveaux d'occupation de la séquence fluviatile. Cet article a pour but de livrer des informations sur la problématique, les stratégies de fouilles et les résultats des différentes études.

\section{LE CONTEXTE STRATIGRAPHIQUE}

Le cadre général stratigraphique de Cagny-l'Épinette, qui appartient à la nappe la plus récente du complexe de la "moyenne" terrasse de la Somme, ne sera que brièvement évoqué car il a déjà été exposé en détail (Antoine et Tuffreau, 1993). La séquence fluviatile se compose d'un cailloutis grossier ( $\mathrm{J}$ : épaisseur $\max .: 1,20 \mathrm{~m}$ ), fortement induré, et de dépôts fluviatiles fins (limons I). Compte tenu du contexte chronostratigraphique des terrasses de la Somme et du caractère cyclique de chacune de ces séquences alluviales qui représente un cycle glaciaire-interglaciaire (Antoine, 1990 ; Hae- saerts et Dupuis, 1986), la nappe de l'Épinette serait attribuable aux stades 10 et 9 des stades isotopiques. Cette hypothèse est en accord avec les résultats d'une datation par E.S.R. effectuée sur les sédiments I qui a donné un âge de $296 \mathrm{ka} \pm 53$ (Laurent, 1993) et les résultats de l'aminostratigraphie (Bates, 1993). La séquence fluviatile fine est particulièrement bien développée dans un chenal (ép. max. : $1,20 \mathrm{~m}$ ) d'orientation sensiblement NW-SE qui, dans le secteur des $\mathrm{m}^{2}$ $25 \mathrm{R}$ à $25 \mathrm{U}$, s'adosse au substrat crayeux qui constituait le versant (fig. 2). Le remplissage de ce chenal débute par un limon fin (12), gris clair (Munsell Soil Color Charts : 10 YR $7 / 2$ ), calcaire, induré (ép. max. : $0,3 \mathrm{~m}$ ), qui apparaît dans la partie nord-ouest de la zone fouillée $\left(m^{2} 21 \mathrm{M}, \mathrm{N}, \mathrm{O}\right.$ et $\left.\mathrm{P}\right)$ (fig. 3).

Le dépôt (I1) qui présente l'extension maximale est un limon gris clair $(2,5$ Y $6 / 2)$ incluant de nombreux petits blocs de craie (1 à $2 \mathrm{~cm})$, des éclats de silex gélivés et des galets tertiaires (ép. max. : 0,60 m). Dans la partie sud du chenal (secteurs des travées 18 à 21 , de $Q$ à $M$ ), les éléments grossiers représentent jusqu'à la moitié de la matrice. II apparaît que cet apport résulte du démantèlement de petits dômes de graviers présents à la partie supérieure du cailloutis comme cela a été nettement observé en 1994 dans la coupe de la travée $25 \mathrm{R}$ à $22 \mathrm{R}$. A proximité du versant crayeux (secteur des $\mathrm{m}^{2} 25 \mathrm{R}$ à $25 \mathrm{U}$ ), la présence de granules de craie, voire de blocs crayeux de dimension décimétrique et de rognons de silex au cortex patiné, résulte d'apports latéraux qui tendent à devenir plus importants que la sédimentation fluviatile fine.

Dans le secteur où le chenal a sa profondeur maximale, un limon (10), gris (10 YR $5 / 2$ à $5 / 3$; ép. max. : $0,35 \mathrm{~m}$ ), à nombreux granules de craie, jalonné à la partie inférieure par un lit brun noir (7,5 YR 3/2), argilo-calcaire organique, et à la partie supérieure par de grosses concrétions calcaires, marquant la formation d'un nouveau chenal incisant le dépôt sous-jacent, apparaît au-dessus du limon I1.

La séquence fluviatile fine se termine par un limon argilo-sableux (I), gris-brun sombre (10 YR 3/3), irrégulièrement présent. Le limon I est souvent érodé par un cailloutis $(\mathrm{H} 1)$ de la base de la couverture, hétérogène (silex épars, granules de craie, concrétions calcaires). La partie inférieure du limon I, qui montre une structure de sol poreuse avec de nombreuses traces de racine et d'oxydations orangées, repose localement $\left(\mathrm{m}^{2} 25 \mathrm{Q}\right.$; campagne de fouilles 1994) sur un plancher calcaire à la surface bosselée (ép. max. : $0,25 \mathrm{~m}$ ). Cet ensemble (l et plancher calcaire) témoigne d'une pédogenèse de type tempéré de zone humide qui se retrouve, latéralement, sur le talus crayeux. Ce dernier est affecté par une altération importante et par des poches de décalcification.

La couverture se compose de dépôts de versant limono-sableux-argileux $(H)$, de sables lités ruisselés (SL), interstratifiés de cailloutis soliflués provenant du versant $(\mathrm{H} 1, \mathrm{G}, \mathrm{F}$, $E 1, E 2)$, qui pourraient correspondre

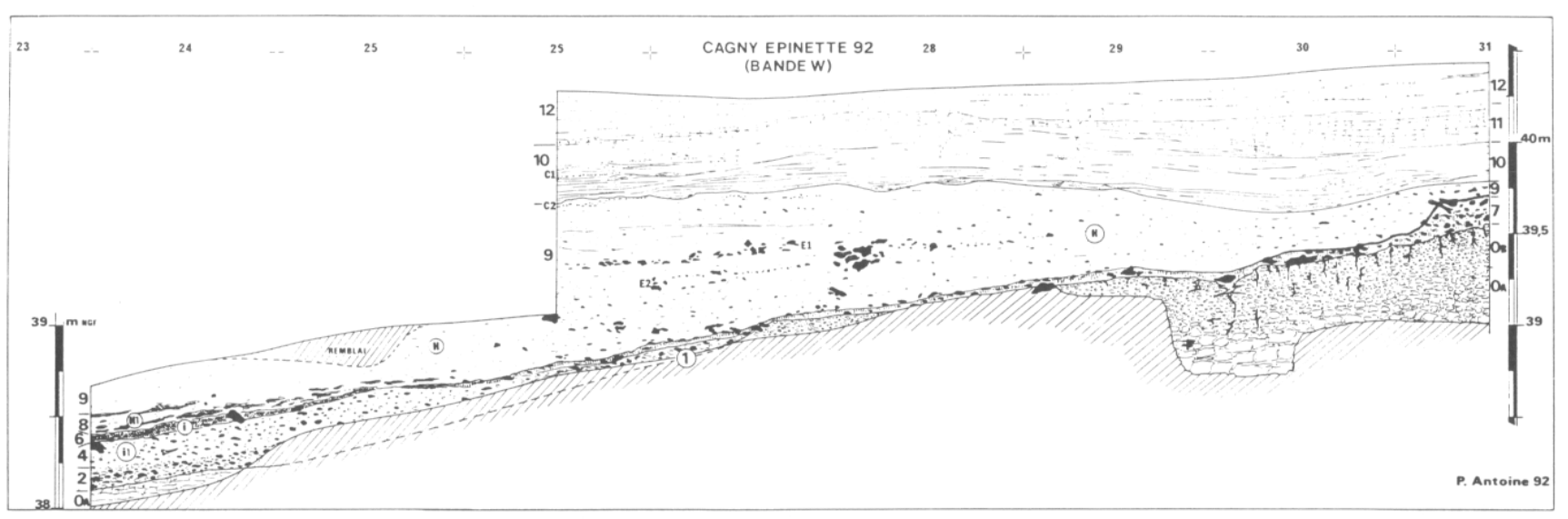

Fig. 2 - Cagny-l'Épinette. Coupe stratigraphique dans la bande W. OA : craie altérée. OB : horizon pédologique développé dans la craie. 1 et 2 : cailloutis de la terrasse $(\mathrm{J}) ; 4$ : limon calcaire gris $(11) ; 6:$ Limon argilo-sableux gris-brun (I) ; $7:$ Cailloutis ; $8:$ Sédiment hétérogène à granules de craie et silex épars; 9 : Base de la séquence de couverture : limon, argilo-sableux $(H)$ et sables lités ruisselés ; 10 : Limon argilo-sableux gleyifié gris ; 11 : Limon argileux, brun jaune, à lits sableux irréguliers, passant à un Bt brun rouge à structure prismatique. 


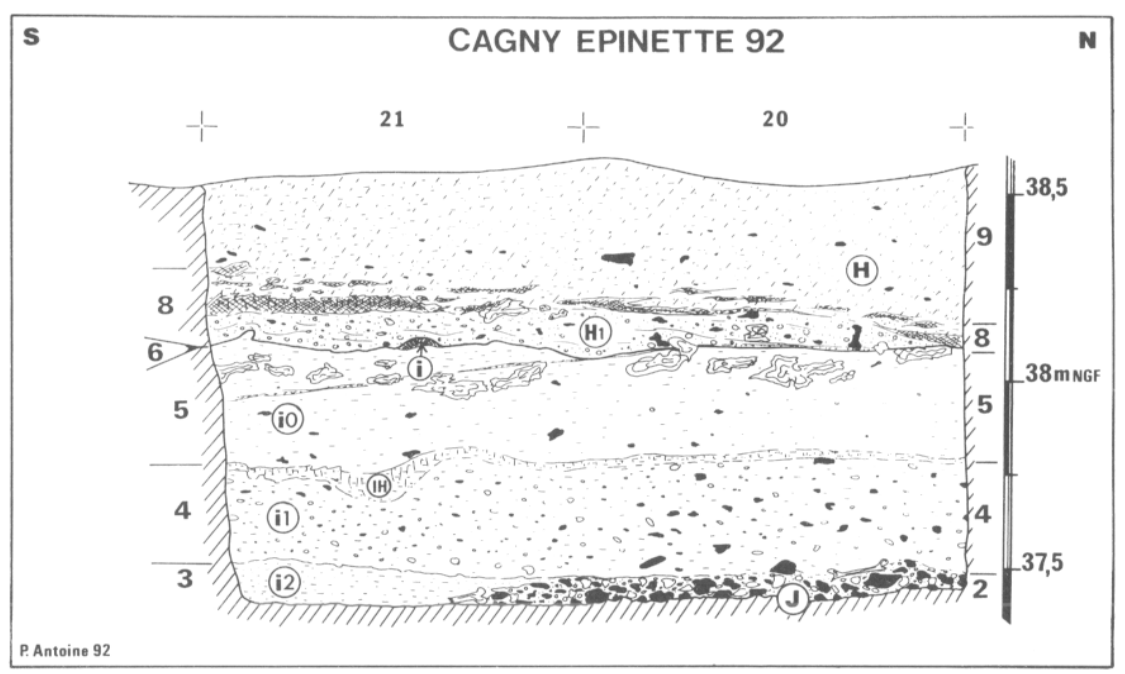

Fig. 3 - Cagny-l'Épinette, coupe détaillée des formations fluviatiles. 2 : cailloutis hétérogène à silex émoussés et galets crayeux $(\mathrm{J}) ; 3$ : Limon fin gris clair (I2) ; 4 : Limon calcaire gris à granules de craie et graviers épars (11); 5 : Limon gris à granules de craie $(10) ; 6$ : Lambeau de limon argilo-sableux gris-brun $(1) ; 8$ : limon hétérogène à granules de craie, silex, concrétions calcaires et lentilles remaniées de l et $10(\mathrm{H} 1) ; 9$ : limon argilo-sableux non calcaire brun gris $(\mathrm{H})$. Granulométrie moyenne des limons fluviatiles 10 , 12 et de la matrice fine de 11 : carbonates $(\mathrm{Caco3})=40$ à $50 \%$; sables $(>50 \mathrm{um})=$ $20-25 \%$; limons $(2-50$ um $)=15-25 \%$; argiles $(<2$ um $)=8-10 \%$.

à une phase de type début glaciaire, ce qui est en accord avec les résultats des analyses palynologiques. Un limon argileux (LAG), gleyifié, gris (10 YR 5/4), à nombreuses traces d'oxydation verticales, jalonné à la base et à la partie supérieure par un cailloutis discontinu ( $\mathrm{C} 2$ et $\mathrm{C} 1$ ), marque une certaine stabilisation du versant. Un sable limoneux, argilifié, affecté par le développement d'un sol lessivé à fragipan, jaune brunâtre (7,5 YR 5/6) apparaît ensuite (B). Cet horizon est sous-jacent à un sable très argileux (A), brun rougeâtre (7,5 YR 5/8), présentant les caractéristiques d'un Bt à forte illuviation, très argileux, compact, où sont observables les extrémités de coins de glaces très effilés. Par ses caractéristiques micromorphologiques (Van Vliet-Lanoë, 1989) et minéralogiques (Balescu, 1988), l'horizon B correspond, d'après la composition en minéraux lourds, au sol SL 2 du "pédocomplexe de Cagny" observable à la partie inférieure des "lœss anciens", d'âge pléistocène moyen, de la grande coupe classique de Cagny-la Garenne (Haesaerts et al., 1984). Cette corrélation est confortée par la composition des minéraux lourds (fig. 4). Latéralement, un sondage ouvert dans l'ancien front d'exploitation, à une trentaine de mètres au nord-ouest de la fouille, a montré la présence d'un vallon colmaté par des limons calcaires à lits de fragments crayeux (LC) qui, par leur fa- ciès et leur contenu minéralogique, pourraient être comparables aux limons calcaires de Cagny-Cimetière, d'âge pléistocène moyen final (étage isotopique 6 ; cf. Haesaerts et al., 1984). L'horizon A peut être attribué au pédocomplexe du dernier interglaciaire (Éémien).

\section{L'ENVIRONNEMENT VÉGÉTAL}

Des analyses palynologiques ont été effectuées en 1980 dans les couches $\mathrm{H}$ et $\mathrm{I}$ dans la partie nordest de la fouille (Munaut, in Tuffreau et al., 1986 ; Munaut, 1988). Aucune évolution sensible n'a été mise en évidence dans la couverture végétale au cours du dépôt sédimentaire qui s'est effectué dans une ambiance forestière (pourcentages des A.P. compris entre $58,8 \%$ et $77,4 \%$ ). Les as- semblages sont dominés par le Bouleau (Betula : $22,6 \%$ ) et le Pin (Pinus : $29,7 \%)$ avec la présence de quelques héliophiles (Artemisia, Composées du type Liguliflore, Chenopodium, Plantago), ce qui indique la présence d'une forêt claire de type boréal. Les pourcentages atteints par les thermophiles, tels que l'Aulne (Alnus : 2 à $8 \%$ ), le Chêne (Quercus : 1 à $8 \%$ ), le Frêne (Fraxinus : $6 \%$ max.), le Noisetier (Corylus : $7 \%$ max.) et la présence discrète du Charme (Carpinus) et du Hêtre (Fagus) indiquent des conditions semblables à celles rencontrées actuellement à la limite de la forêt boréale.

De nouveaux échantillons ont été collectés en 1991 dans la partie est de la fouille où la séquence fluviatile s'épaissit dans le remplissage d'un chenal (colonne II).

La colonne III donne une succession qui peut s'interpréter de la manière suivante : steppe légèrement arborée (I1), sylvosteppe tempérée (I), retour vers un paysage ouvert où subsistent des arbres tempérés et modérément steppiques $(\mathrm{H})$. Si on admet que la sylvosteppe tempérée est une expression des interglaciaires du Pléistocène moyen on aurait une succession du type Tardiglaciaire (I1), Interglaciaire (I) et Début Glaciaire $(H)$. Malgré le faible nombre de pollens, il n'y a pas impossibilité de synchroniser le contenu de la couche 11 de la colonne I avec le contenu de la couche 11 de la coIonne III. La couche 10 pourrait constituer un ensemble intermédiaire entre les couches II et I, c'est-à-dire le début de l'Interglaciaire caractérisé par un recul de la steppe et l'avancée des arbres tempérés. La colonne II débute dans la couche 12 par une forêt claire boréale, parsemée d'arbres tempérés, assez proche sans doute de la limite sud de ce biome tel qu'il pourrait se pré-

Tabl. 1 - Résultats des analyses palynologiques de Cagny-l'Épinette.

\begin{tabular}{|l|c|c|c|c|c|c|c|}
\hline & couche & $\begin{array}{c}\text { Nbre } \\
\text { pollens }\end{array}$ & AP & Boréaux & Tempérés & Steppiques & Aquatiques \\
\hline colonne III & $\mathrm{H}$ & 142 & 36,5 & 23,2 & 13,3 & 33,2 & - \\
$\mathrm{m}^{2}: 23 \mathrm{X}$ & $\mathrm{I}$ & 520 & 72,0 & 38,8 & 33,2 & 19,3 & 0,8 \\
& $\mathrm{I}$ & 200 & 24,0 & 20,0 & 3,1 & 74,0 & - \\
colonne I & $I 0$ & 126 & 38,1 & 26,0 & 10,4 & 54,0 & - \\
$\mathrm{m}^{2}: 21 \mathrm{U}$ & $\mathrm{I}$ & 14 & 28,5 & 28,5 & - & 53,5 & - \\
& $\mathrm{IO}+\mathrm{I1}$ & 140 & 37,1 & 27,1 & 10,0 & 55,0 & - \\
colonne II & $I 0$ & 588 & 54,9 & 44,5 & 10,4 & 43,9 & 0,2 \\
$\mathrm{~m}^{2}: 21 \mathrm{P}$ & $\mathrm{I}$ & 402 & 54,8 & 38,7 & 15,9 & 42,6 & - \\
& $I 2$ & 312 & 74,7 & 65,4 & 9,3 & 23,4 & - \\
\hline
\end{tabular}


Fig. 4 - Corrélations stratigraphiques des séquences de Cagny-la Garenne et de Cagny-l'Epinette sur base stratigraphique, micromorphologique, minéralogique et TL relative (d'après Antoine, 1990 ; Antoine et Tuffreau, 1993 ; Haesaerts et al., 1984 ; Haesaerts et Dupuis, 1986 ; Balescu, 1988 et Van Vliet-Lanöe, 1989). BTS : horizon BT du sol de surface; LR : lœss weichséliens; $\mathrm{SH}$ : complexe de sols humifères (Début Glaciaire Weichsélien) ; $F$ : fentes de gel à remplissage argilo-organique (ouverture au sommet de SH) ; SR/A : horizon BT sur loess (sol de Rocourt, Éémien) ; LAC/LC : loess calcaires à granules de craie ; LAS : lœss sableux non calcaires ; SL2/GSL3/SL3 (PCC) : pédocomplexe de Cagny comportant deux horizons BT (SL2 et SL3) séparés par un dépôt caillouteux soliflué (GSL3) ; GLS/SL : sables limoneux à lentilles de graviers argileux solifluées; $E / F / G / H / L A G$ : dépôts de pente sablo-limoneux ruisselés à lentilles de cailloutis solifluées ; ARB : paléosol argileux développé au sommet de PR ; PR : coulée de craie gélifluée; SLL : sables et limons lités de la Garenne ; SV : sables verts fluviatiles ; 1 : dépôts fluviatiles fins de l'Épinette avec petit sol de milieu humide au sommet ; GF': graviers fluviatiles de la Garenne ; DVC : ensemble de dépôt de versant crayeux à silex à lentilles fluviatiles interstratifiées ; $\mathrm{J}$ : cailloutis fluviatile de l'Épinette ; $\mathrm{CR}$ : substratum. alt. rel. : altitude relative par rapport au creusement maximum de la vallée.

senter au début ou à la fin d'un interglaciaire, ou durant un interstade tempéré. La présence non négligeable de Picea, taxon qui apparaît en fin d'Interglaciaire, fait pencher la balance en faveur de l'hypothèse fin d'Interglaciaire.

La couche 11 de la colonne II montre une avancée notable des plantes steppiques aux dépens de la forêt boréale, mais les arbres tempérés progressent un peu. On peut y voir le signe d'une continentalisation plutôt que d'un refroidissement. La couche 10 lui est pratiquement semblable et n'indique pas de changement dans le paysage. On remarque la présence d'arbres tempérés de fin d'Interglaciaire (Carpinus, Fagus, Picea). S'il n'existe pas d'hiatus tem- porel important entre ces trois couches du profil, on assiste à une évolution normale correspondant à une fin d'Interglaciaire ou à un Début glaciaire.

Par contre, le contenu moyen de la couche 10 du profil II n'est pas semblable à celui du profil I qui luimême montre un paysage nettement plus continental bien qu'il y ait continuité stratigraphique entre 10 de la colonne I et 10 de la colonne II.

II apparaît donc que la couche I du profil III représente un Interglaciaire. Cette interprétation est en accord avec les données stratigraphiques qui permettent d'attribuer la pédogenèse de la couche l et le concrétionnement sous-jacent ob- servé lors de la campagne 1994 à un épisode tempéré de type interglaciaire ou à un épisode de début d'interglaciaire pollué par quelques pollens de type Picea et Fagus. La couche 12 du profil II pourrait correspondre à un épisode du Début Glaciaire précédant piégé dans un ravinement de la partie externe de la nappe de graviers de l'Épinette.

La couche I serait donc un dépôt fluviatile, présentant une pédogenèse, de l'étage isotopique 9 , correspondant dans la biostratigraphie du bassin Nord de la Mer du Nord à l'Interglaciaire Holsteinien.

La couche 12 et les graviers sousjacents de la partie externe de la nappe de l'Épinette se seraient déposés durant le Début Glaciaire de l'étage isotopique 10 , c'est-à-dire durant le début du Glaciaire correspondant dans la biostratigraphie du Nord à l'Elstérien. Une situation comparable a pu être observée à Saint-Sauveur (Antoine et al., 1994) où le "Sol gris de Saint-Sauveur", corrélé avec le Sol SS1 de SaintSauflieu (Début Glaciaire Weichsélien), repose de façon analogue sur la nappe d'Étouvie, après le dépôt local d'une lentille fluviatile appartenant au même stade froid que celle-ci. Le pléniglaciaire de l'étage 10 serait représenté par les graviers non calcaires qui ont été observés en 1990 dans un sondage ouvert à une vingtaine de mètres au nord-ouest de la fouille, à la limite de l'ancien front d'exploitation de la carrière. Cette interprétation suppose la présence d'un bilan comparable à ce que l'on observe dans la nappe de la $\mathrm{Ga}$ renne (Antoine, 1990 ; Antoine et Tuffreau, 1993). Le contact entre, d'une part, les graviers du secteur de la fouille et la lentille 12 (Début Glaciaire) et, d'autre part, le limon 11 (Tardiglaciaire) correspondrait à un hiatus très important.

Si l'on admet que la succession I2, I1, 10 du profil II correspond à un Début Glaciaire, il faut faire intervenir la présence d'un biseau dans 11, entre la partie la plus profonde du chenal (colonnes II et I) et la partie du chenal moins épaisse où seul I1 est présent. Dans ce cas, il conviendrait de différencier deux unités stratigraphiques différentes dans la couche 11, ce qui n'a pas été observé sur le terrain. II faut toutefois noter que la couche 11 comprend beaucoup d'éléments grossiers dans la partie ouest du chenal, la plus pro- 
fonde, alors que les éléments crayeux sont plus nombreux ailleurs (cf. supra) ce qui pourrait plaider en faveur de la présence de deux unités stratigraphiques dans I1. L'étude de la répartition spatiale des fragments osseux, notamment, celle, en cours, des remontages et des appariements est susceptible d'apporter des éléments de réponse permettant de savoir si I1 correspond à deux unités stratigraphiques distinctes, une remontant à une phase de type début glaciaire, l'autre à une phase de type tardiglaciaire ou si les quelques pollens d'espèces thermophiles du profil II sont présents à l'état de reliques.

D'un point de vue palynologique, il est important d'insister sur le fait que contrairement à l'Holocène dont les dépôts, dans le bassin de Somme, sont tourbeux et le déroulement est comparable à ceux du Nord du Bassin de la Mer du Nord, tous les autres dépôts interglaciaires et interstadiaires observés dans le Nord du Bassin Parisien sont d'un type différent, caractérisé par un élément plus continental, sans sédiments organiques, et dans un milieu de sylvosteppe.

\section{UES MICROMAMMIFÈRES}

Des échantillons de sédiment ont été prélevés à Cagny-l'Épinette en 1992 et 1993 dans le but de rechercher des restes de micromammifères fossiles. De volumineux échantillons ont été pris dans les couches $\mathrm{H}$ et $\mathrm{I}$. Une étude préliminaire a montré que la couche $\mathrm{H}$ est plutôt pauvre. En raison d'une décalcification, la couche $\mathrm{H}$ a livré un petit nombre de restes mal conservés de Talpa europaea, Arvicola sp. et Microtus sp. Les restes de la couche I1 sont bien conservés et nombreux. Les groupes et espèces suivants ont pu être identifiés : poissons ; amphibiens : crapaux et/ou grenouilles ; mammifères : Sorex sp. (Sorex araneus), Talpa europaea, Arvicola terrestris, Microtus arvalis/Microtus agrestis, Microtus œconomus. la liste des espèces diffère de celle présentée par Cordy (1989). Neomys sp., Microtus gregalis et Pitymys subterraneus n'étaient pas représentés dans notre prélèvement. Les micromammifères de la couche I sont dominés par le Campagnol des champs et/ou par le Campagnol agreste Microtus arvalis/M. agrestis, espèces qui sont ca- ractéristiques d'un milieu restreint à ouvert. La Musaraigne Sorex sp., morphologiquement similaire à la musaraigne commune Sorex araneus, le rat taupier Anvicola terrestris et le Campagnol nordique Microtus œconomus (Microtus cf. malei) sont bien représentés. Le Rat d'eau taupier est semi-aquatique et le campagnol nordique préfère les conditions humides. La Taupe Talpa europaea est rarement représentée dans la faune. Cette espèce, fréquentant originellement un paysage de forêt à espèces caduques (Corbet et Harris, 1991), est maintenant présente dans la plupart des endroits où le sol est suffisamment humide pour permettre de faire des tunnels. Elle est peu fréquente dans les forêts de conifères.

Les espèces caractéristiques d'un environnement boisé telles que le Campagnol roussâtre Clethrionomys glareolus et les loirs sont absentes. II en est de même pour les micromammifères tels que les spermophiles et les hamsters qui témoignent de conditions steppiques plus continentales et pour les espèces comme les lemmings qui vivent dans des conditions arctiques. On peut donc établir que la faune de petits mammifères de la couche I1 de Cagny-l'Épinette correspond à un environnement ouvert et à des conditions climatiques tempérées fraîches, c'est-à-dire ni glaciaires ni interglaciaires. II s'agit des conditions climatiques qui ont probablement dominé dans toute l'Europe du Nord-Ouest au cours des derniers 500000 ans.

La faune de la couche I1 de Cagnyl'Épinette est plutôt moderne. Des espèces telles que Sorex (Drepanosorex) sp., Talpa minor, Trogontherium cuvieri et Pliomys episcopalis, qui apparaissent dans les assemblages du Cromérien récent de $\mathrm{Mi}$ senheim I (Allemagne), Boxgrove (Angleterre) et de la Belle Roche à Sprimont (Belgique) et qui sont considérées comme étant des reliques du Pléistocène moyen ancien, sont absentes. Le plus grand Rat taupier Arvicola terrestris de l'Europe du Nord-Ouest et de l'Europe centrale montre une évolution plutôt rapide durant la seconde moitié du Pléistocène moyen. Cette évolution se manifesta par un développement différent de la bande d'émail qui recouvre les triangles de dentine. Heinrich $(1978,1987)$ a développé une méthode (S.D.Q.) pour calculer les différences concernant la bande d'émail. L'épaisseur de l'émail sur les deux côtés des angles saillants de la $M_{1}$ est mesurée. L'épaisseur de la bande d'émail postérieure x 100 est divisée par celle de la bande d'émail antérieure. La valeur S.D.Q. de chaque M1 est la moyenne des valeurs S.D.Q. de tous les angles saillants. Cette méthode a été, depuis, employée par beaucoup d'auteurs (Kolfschoten, 1990) et les données concernant le stade d'évolution du Rat taupier fossile ont été souvent utilisées pour situer les faunes dans un ordre biostratigraphique.

La valeur du S.D.Q. montre un déclin graduel dans le temps (Heinrich, 1982 ; Kolfschoten, 1990) qui est cependant interrompu par une importante fluctuation à la transition Saalien-Éémien. L'étude des données concernant Arvicola terrestris dans le Nord-Ouest de l'Europe indique, durant l'Éémien, une immigration de populations moins dérivées en provenance du Sud-Est de l'Europe après la disparition des populations locales en raison de l'avancée du glacier. Cette nouvelle immigration entraîna une fluctuation dans la courbe générale de l'évolution, ce qui limite les applications pour la biostratigraphie.

La valeur de l'indice S.D.Q. pour les échantillons de Cagny-l'Épinette est d'environ 100 (fig. 5). Des valeurs similaires sont connues pour les faunes du début Saalien de Maastricht-Belvédère 4 (horizon archéologique principal), WageningenFransche Kamp et WeimarEhringsdorf (Kolfshchoten, 1990). Elles sont plus faibles que celles des populations d'Arvicola de Neede (Pays-Bas), d'âge holsteinien (Kolfschoten, 1990) et de Schöningen 12 B (Allemagne) d'âge post-elstérien et plus probablement post-holsteinien, c'est-à-dire début saalien (Thieme et al., 1993). D'autre part, elles sont plus faibles que celles de Schweinskopf-Karmelenberg et de Wannen (Allemagne) d'âge saalien récent (étage isotopique 6 ; cf. Kolfschoten et Roth, sous presse).

Une comparaison des valeurs de S.D.Q. d'Arvicola de Cagny-l'Épinette (niveau I1) et d'autres populations du Pléistocène moyen montre que les faunes de Cagny-l'Épinette sont proches de celles de Maastricht-Belvédère 4 et de Weimar-Ehringsdorf qui sont corrélées avec le Saalien ancien et attribuées à l'étage isotopique 7 d'après les méthodes de datation absolue. 
Plaidter Hummerich $\mathrm{A}(\mathrm{N}=12)$

Wannen $(\mathrm{N}=2)$

Rhenen ( $\mathrm{N}=9)$

Maastricht-Belvédère-4 $(\mathrm{N}=25)$

Weimar-Ehringsdorf $(\mathrm{N}=7)$

CAGNY L'EPINETTE $(N=7)$

Ariendorf $1(\mathrm{~N}=4)$

Schöningen 12B $(\mathrm{N}=15)$

Bilzingsleben $(\mathrm{N}=5)$

Neede $(\mathrm{N}=12)$
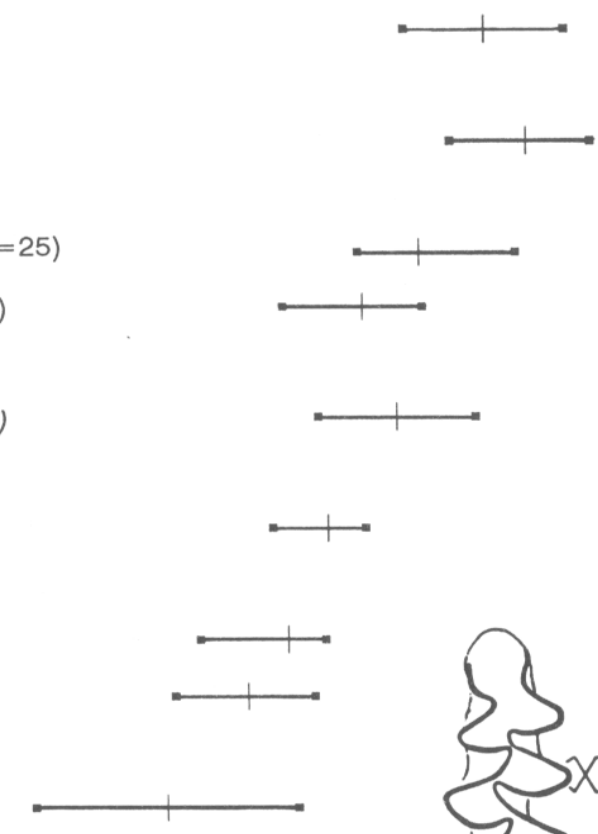

(1)

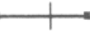

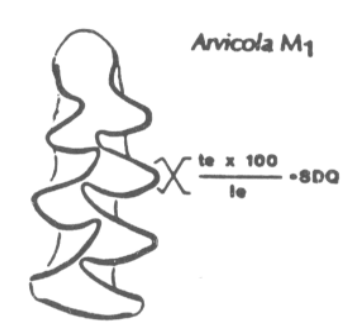

Miesenheim I $(\mathrm{N}=78)$

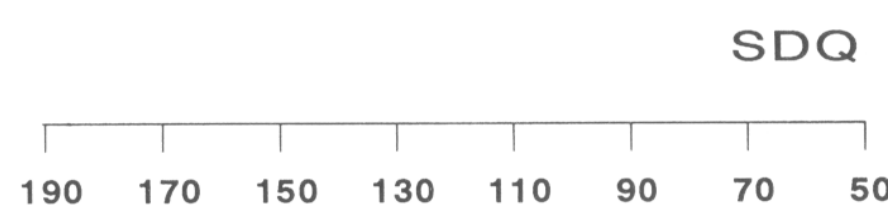

Fig. 5 - Cagny-l'Épinette. Indices de SDQ d'Arvicola du niveau I de Cagny-l'Épinette par rapport à ceux de populations d'autres gisements du Pléistocène moyen.

\section{STRATÉGIES DE FOUILLES}

Depuis 1989, les campagnes de fouilles ont surtout concerné les niveaux 11, qui à partir de 1993 a été subdivisé en 11 et $11 \mathrm{~b}$. De nombreuses raisons permettent de penser qu'il s'agit de niveaux correspondant à une surface d'occupation unique ou d'occupations qui ont été réduites en nombre. Tout d'abord, le contexte sédimentaire est favorable en raison de la faible énergie de la dynamique fluviatile. Ensuite, la disposition du matériel archéologique correspond à une concentration assez dense, peu épaisse et relativement horizontale, ce qui signifie qu'il fut abandonné sur une surface assez régulière. Sa nature plaide également en ce sens. II s'agit de concentrations de silex allant des petits éclats et débris à de gros rognons et d'ossements et de fragments comprenant toutes les catégories depuis des petites esquilles jusqu'à des os- sements complets de grands herbivores (cervidés, équidés et bovidés), sans évidence d'un classement résultant d'une action de la rivière. Enfin, les observations sur le terrain et l'examen des premiers plans de répartition permettent de suggérer que la répartition du matériel archéologique n'est pas aléatoire.

Cependant, des arguments en faveur de l'existence, au Paléolithique inférieur, de sols d'habitat dont la reconnaissance découle de données comparables ont été fortement critiqués. Dans un certain nombre de cas, il a pu être démontré que des facteurs non anthropiques ont été la cause des dispositions observées pour trois raisons, plus particulièrement, dans le cas des gisements du Paléolithique inférieur. La probabilité de l'action de phénomènes taphonomiques est importante en raison de leur très grande ancienneté. La faiblesse de l'organisation des traces d'occupations au Paléolithique infé- rieur rend les structures évidentes et les structures latentes rares, contrairement à ce que l'on observe dans les périodes plus récentes, ce qui constitue un handicap pour les analyses spatiales. Le caractère "moderne" des populations du Paléolithique inférieur et moyen est l'objet d'un grand débat (Chase et Dibble, 1987 ; Mellars, 1988, 1991) qui est en partie alimenté par la présence ou l'absence de sols d'habitat durant les périodes concernées. Les arguments invoqués concernent ceux relatifs à l'organisation spatiale et aux liens fonctionnels entre les activités, l'existence de camps de base, l'aménagement d'abris et de pavement, le transport et le stockage de nourriture, la chasse, la consommation de la viande, les différences (organisation spatiale, dimensions) entre les gisements du Paléolithique inférieur et moyen et ceux du Paléolithique supérieur (Barral et Simone, 1972 ; Bonifay, 1976 ; Bunn, 1981, 1983 ; Bunn et Kroll, 1986 ; Freeman, 1975, 1978 ; Isaac, 1983, 1984 ; Kind, 1985 ; Leroi-Gourhan, 1961 ; Lovejoy, 1981 ; Lumley, 1969 ; Lumley et Boone, 1976 ; Mania et Weber, 1986, 1966 ; Potts, 1984 ; Simek, 1987 ; Stekelis et al., 1969 ; Tuffreau et Sommé, 1988).

L'importance des implications qui sont en jeu nécessite de tester les hypothèses favorables ou défavorables concernant la réalité de ce qui peut s'apparenter à des sols d'habitat lors de la fouille, ce qu'il serait impossible de faire après. L'analyse critique de la nature du niveau 11 a influencé à la fois les stratégies de fouilles et celles de l'analyse.

Les techniques de fouilles employées sont celles utilisées dans les gisements susceptibles de contenir des sols d'habitat (Leroi-Gourhan et Brézillon, 1972). Les artefacts sont dégagés de leurs sédiments et laissés en place sur une superficie couvrant plusieurs $\mathrm{m}^{2}$. Le secteur dégagé fait l'objet de couvertures photographiques verticales, de dessins. La position de chaque pièce est enregistrée avec un théodolite électronique. Cette méthode d'enregistrement permet de réunir les données nécessaires à l'analyse d'un sol d'habitat mais n'est pas suffisante pour en démontrer la réalité.

C'est pourquoi, un certain nombre d'ajouts dans les méthodes de fouilles ont été apportés afin de collecter des données appropriées à la problématique et de répondre à plu- 
sieurs types de questions, c'est-àdire l'importance de l'action des agents naturels (transport et classement du matériel par le cours d'eau et l'action des carnivores, l'intégrité de l'assemblage en terme de comportement (éventuelle exportation de supports et d'outils ou mélange résultant de plusieurs occupations), liens ou absence de liens entre la faune et les éléments lithiques (Chase et al., 1994).

Les remontages bien que n'étant une panacée (Bordes, $1980 \mathrm{a}$ et b) sont nécessaires pour apprécier la réalité de la surface de répartition des ossements. Dans le cas présent, ceux concernant les ossements sont plus susceptibles d'apporter des informations (Enloe et David, 1989) car la viande pourrit rapidement alors que les matériaux lithiques peuvent être réutilisés de multiples fois. Cependant, l'analyse de leur répartition horizontale et de leurs remontages (Cahen et al., 1979) peut apporter des éléments à l'encontre d'une action de la dynamique fluviatile alors qu'une distribution verticale indique un remaniement (Villa, 1982, 1983). Cependant, il faut garder à l'esprit que les causes de remaniement sont très nombreuses et qu'il est difficile de les déceler sur le terrain (Hofman, 1992).

L'enregistrement de l'orientation des artefacts est un moyen de déceler une éventuelle influence de la dynamique fluviatile dans la constitution de l'assemblage ou dans un déplacement des pièces. L'orientation horizontale des artefacts est susceptible de détecter l'influence du cours d'eau qui dispose les pièces parallèlement ou perpendiculairement à son action (Isaac, 1967 ; Kelling et Williams, 1967 ; Kluskens, sous presse). En outre, l'orientation verticale des artefacts déposés sur une surface dépend de sa topographie alors que celle des pièces déplacées par piétinement ou par bioturbation est plus aléatoire. L'action du courant tend aussi à enfoncer l'extrémité des pièces en enlevant du sédiment fin qui est redéposé en arrière. Le fait que les éclats soient trouvés reposant sur la face inférieure ou sur la face supérieure est également significative.

Le tamisage à l'eau est utilisé pour retrouver de très petits artefacts et fragments d'ossements. Le classement par dimension est important pour reconnaître de multiples sortes de perturbations, notamment celles dues à l'eau. Une concentration de grands artefacts alors que les éléments petits sont rares, et viceversa, peut être une indication significative d'un remaniement. Par contre, la production d'outils et leur réavivage, attestés par la présence d'éclats de retouche et de préparation est un critère permettant de reconnaître la présence d'une surface d'occupation où des activités de maintenance ont eu lieu.

Les analyses géophysiques, essentiellement la susceptibilité magnétique et la résistivité électrique, sont utilisées pour déceler les secteurs perturbés ou les aires de combustion qui seraient autrement indécelables.

\section{MÉTHODE D'ENREGISTREMENT INFORMATISÉ}

Jusqu'en 1991, les surfaces dégagées sur plusieurs $\mathrm{m}^{2}$ faisaient l'objet de différentes méthodes d'enregistrement des artefacts avant qu'ils ne soient retirés : dessin à la main, couverture photographique verticale par $\mathrm{m}^{2}$ avec un appareil de moyen format, coordonnées $X, Y$ et $Z$ pour chaque pièce, orientation (horizontale, inclinée et verticale) de l'axe longitudinal et de la largeur des pièces allongées.

A partir de 1991, un certain nombre de méthodes d'enregistrement furent adoptées de façon à pouvoir mieux prendre en compte les aspects taphonomiques de la surface d'occupation supposée du niveau I1. Un théodolite électronique est connecté à un micro-ordinateur et à une imprimante portable. II est ainsi possible d'enregistrer la provenance de tous les objets et d'imprimer des étiquettes qui sont incluses dans chaque sachet avec les pièces. Ce système qui a été élaboré pour les fouilles de La Quina et de Combe-Capelle bas a déjà été publié (Dibble, 1987 ; Dibble et McPherron, 1988). Les implications de l'adoption de cette méthode sont nombreuses. La précision de l'enregistrement en trois dimensions des artefacts est plus grande, ce qui permet un

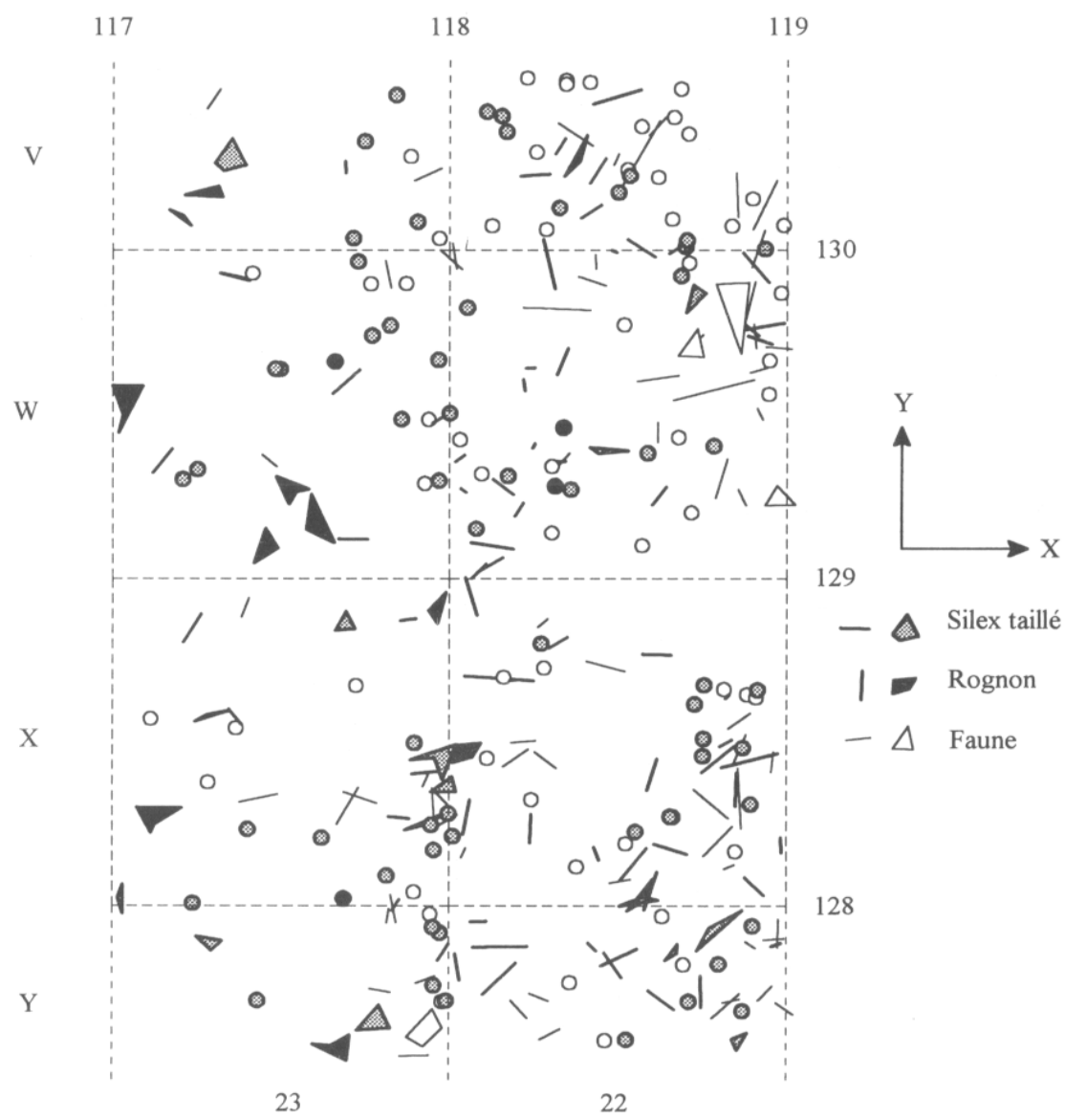

Fig. 6 - Cagny-l'Épinette. Plan de répartition des vestiges d'un secteur du niveau 11, établi à partir d'un enregistrement avec un théodolite électronique. 


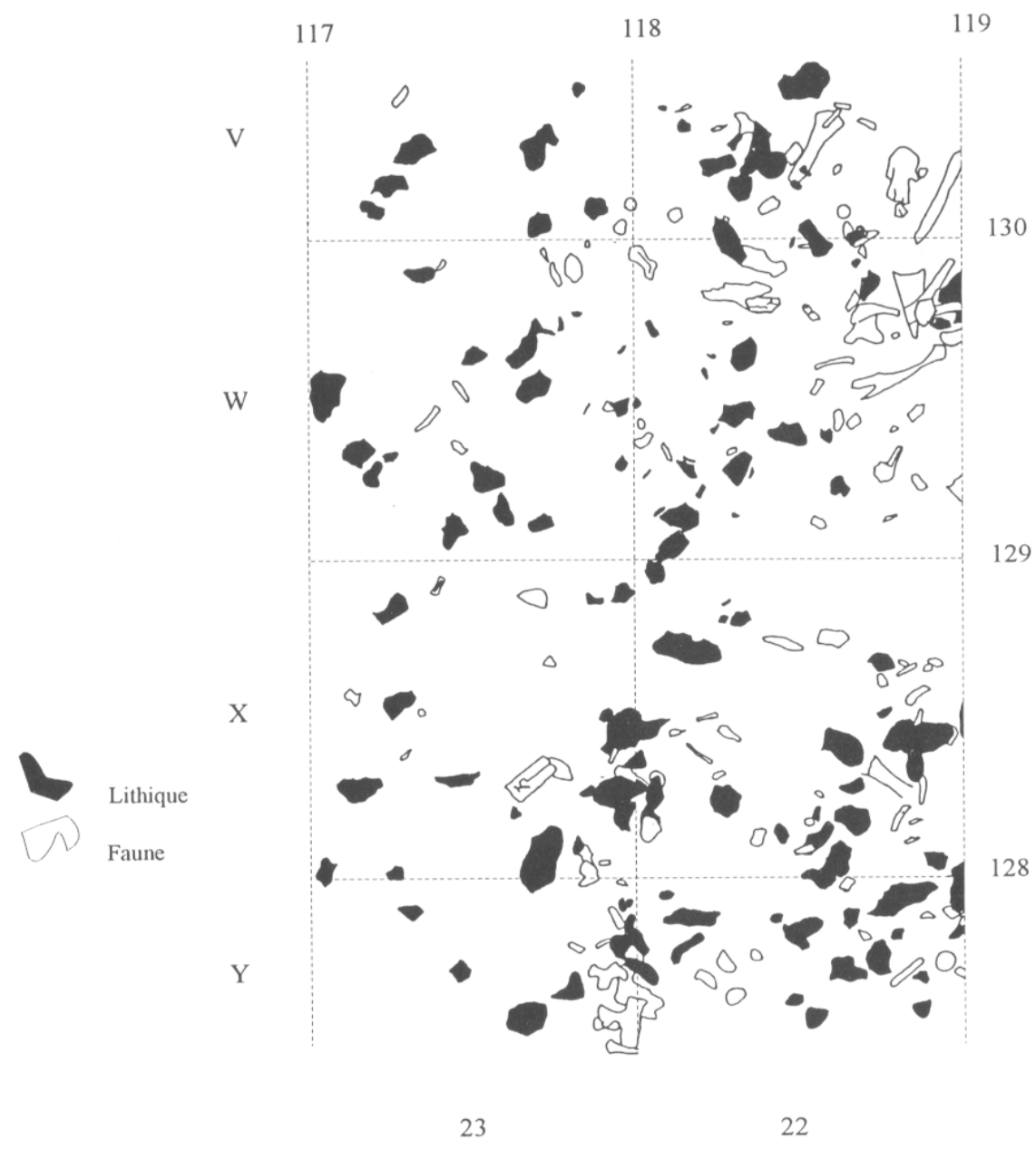

Fig. 7 - Cagny-l'Épinette. Niveau I1. Plan du même secteur que celui de la fig. 6 tel qu'il apparaît après de levés manuels.

contrôle plus précis de la répartition horizontale et verticale des objets (fig. 6). Les erreurs inhérentes avec une entrée manuelle des données sont éliminées. L'analyse de la répartition horizontale et verticale de catégories variées d'artefacts est rendue possible en peu de temps, avec une précision que ne saurait atteindre des plans faits à la main, ce qui n'empêche toutefois pas la prise de photographies verticales et la réalisation de dessins sur le terrain (fig. 7).

Enfin, l'orientation des artefacts est automatiquement prise en compte avec l'enregistrement de deux points à chaque extrémité du grand axe pour toutes les pièces allongées.

\section{EXTENSION DES NIVEAUX ARCHÉOLOGIQUES}

La couverture sablo-limoneuse (niveaux archéologiques $H$ à $C 1$ ) est présente sur tout le secteur concerné par la fouille. Par contre, l'extension des limons de la séquence fluviatile faible. Le niveau I est visible sur la sent partout). majeure partie de la zone fouillée. Dans la partie ouest, il a été érodé par le cailloutis $\mathrm{H} 1$ localement observable à la base du limon sablo-argileux $\mathrm{H}$. Le niveau 10 , qui correspond à un limon remplissant un petit chenal incisant le chenal principal n'est visible que dans le secteur où ce dernier a son épaisseur maximale (partie ouest de la fouille). L'extension du niveau 11 coïncide avec celle du chenal. Le niveau 12 a été reconnu sur une superficie de 5 à $6 \mathrm{~m}^{2}$.

Dans la partie est de la fouille, où le chenal est colmaté par du sédiment fluviatile fin, le limon argileux gris-brun I repose directement sur les graviers $(J)$ de la terrasse. Des silex taillés et des fragments d'ossements d'animaux sont présents à la partie inférieure du limon I et parfois reposent directement sur la surface du cailloutis grossier J. Ce niveau archéologique a été dénommé I-J. Les vestiges lithiques et osseux se répartissent de façon assez diffuse dans le niveau l-J (Tuffreau et al., 1986). La partie supérieure des graviers de la terrasse (J) a été fouillée sur quelques $\mathrm{m}^{2}$ (fig. 8 ).

\section{LES INDUSTRIES LITHIQUES}

Les industries lithiques de Cagnyl'Épinette sont incluses dans les cailloutis de la couverture sablolimoneuse ou proviennent des différents niveaux archéologiques de la séquence fluviatile fine. La matière première utilisée est constituée de rognons de silex locaux, de qualité le plus souvent médiocre, présentant un cortex usé par la rivière. II s'agit

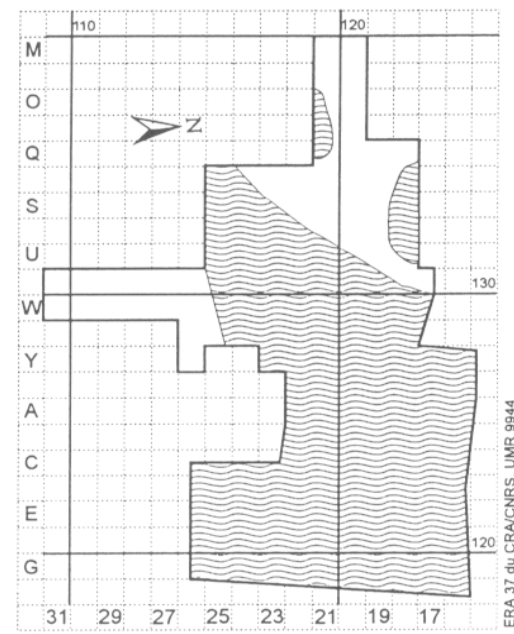

Fig. 8 - Cagny-l'Épinette. Extension des niveaux archéologiques. 1 : limites de la fouille en $1993 ; 2$ : niveau $12 ; 3$ : niveau $10 ; 4$ : niveau $11 ; 5$ : niveau $1 ; 6$ : niveau $\mathrm{H}$ (pré- 
de rognons provenant de la nappe fluviatile de l'Épinette, ou d'apports latéraux sur le versant, constitués de nappes fluviatiles, plus anciennes, démantelées. D'une manière générale, les industries lithiques se caractérisent par la présence d'un nombre plus ou moins élevé de bifaces, de morphologie variable, ayant souvent gardé une base corticale importante. De nombreux rognons de silex présentent un tranchant à l'extrémité distale obtenu par une ou deux séries d'enlèvements permettant de les assimiler à des chopping-tools. Le débitage Levallois est quasiment absent. Les nucléus, qui ont souvent gardé d'importantes plages corticales, ont été faiblement exploités. lis présentent peu de surfaces de débitage. La gestion du débitage est fréquemment unipolaire ou bipolaire. Les outils sur éclats sont souvent réalisés sur des éclats corticaux, et dans de nombreux cas sur des gélifracts. Les encoches et les denticulés constituent toujours la catégorie la mieux représentée. Les racloirs présentent souvent des tranchants discontinus aux retouches assez irrégulières ce qui les rend parfois difficiles à différencier des éclats à retouches irrégulières qui sont toujours bien représentés. Les outils de type paléolithique supérieur sont rares mais toujours présents. II s'agit surtout de grattoirs et de couteaux à dos retouché de mauvaise facture.

Deux séries lithiques, celle de $\mathrm{H}$, étudiée par A. Lamotte et celle de I1, analysée par $H$. Dibble, serviront à illustrer les caractéristiques des industries de Cagny-l'Épinette.

\section{- L'industrie lithique du niveau $H$}

L'industrie lithique du niveau $\mathrm{H}$, provenant des campagnes de fouilles de 1980 à 1991, se compose de 1089 artefacts. II s'agit d'une série fraîche, sans éléments roulés et à rares pièces patinées (fig. 9). Cette série est caractérisée par une très faible présence des outils sur blocs (tabl. 2). Aucun chopping-tool n'a été décompté et l'ensemble est constitué en proportion quasi-égale de bifaces et de choppers. La série est dominée par la catégorie des éclats et des fragments d'éclats. A l'intérieur de celle-ci, les vestiges de moins de $20 \mathrm{~mm}$ sont rares. A l'opposé, les fragments d'éclats de plus de $20 \mathrm{~mm}$ sont particulièrement bien représentés. II convient de noter l'im-

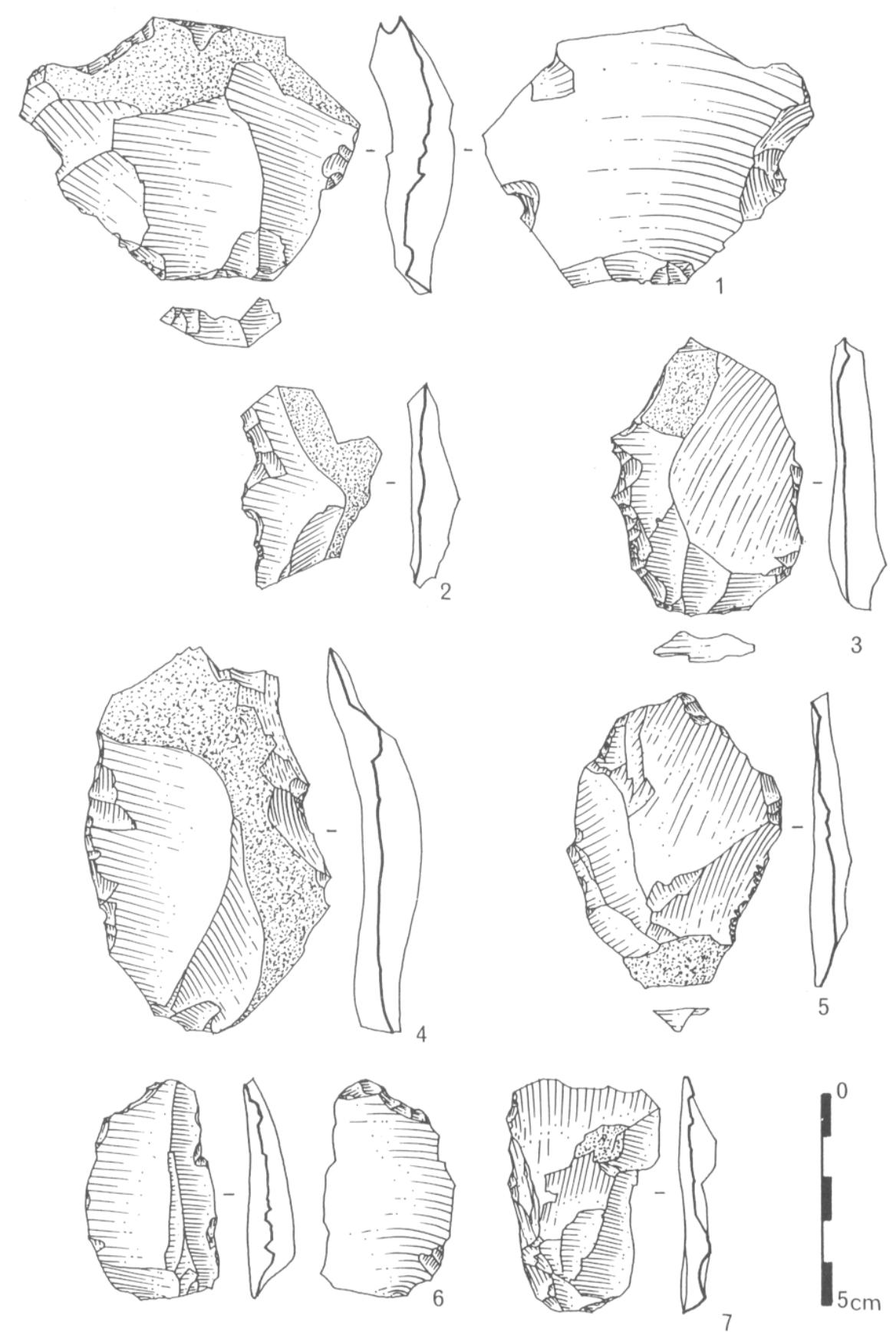

Fig. 9 - Cagny-l'Épinette, niveau $\mathbf{H}$, industrie lithique. 1 : outil composite ; 2 : denticulé ; 3 et 4 : racloirs ; 5 : retouches irrégulières ; 6 : éclat tronqué ; 7 : éclat de taille de biface.

portance quantitative des éclats fracturés en partie distale $(n=82$ ) ou latérale $(n=26)$, soit $26 \%$ des éclats. Les éclats débordants $(n=9)$ attestent le débitage de même qu'un ravivage des nucléus sur place. Les divers indices typologiques et technologiques sont en général très faibles, plus particulièrement l'indice des bifaces et celui de l'acheuléen uniface (tabl. 2 et 3). L'importance relative de ceux des encoches et des denticulés est due au très faible nombre des racloirs. II est en effet très difficile de différencier ces outils des éclats à retouches irrégulières en raison de la faible profondeur des tranchants. II en est de même pour certaines encoches très larges. II ne convient donc de ne pas accorder trop d'importance à la signification réelle des indices des outils sur éclat pour une industrie dont l'outillage est aussi peu standardisé.

\section{- ÉtUde teCHNOLOGIQUe}

\section{- Les produits de débitage}

Avec $81 \%$ des éclats dont la longueur est comprise entre 20 et $60 \mathrm{~mm}, 80 \%$ des éclats de largeur 
Tabl. 2 - Décompte général de la série H (Cagny-l'Épinette).

\begin{tabular}{|l|r|}
\hline & Nombre \\
\hline Bifaces & 11 \\
Choppers & 4 \\
Encoches & 172 \\
Denticulés & 38 \\
Racloirs droits & 10 \\
Racloirs convexes & 3 \\
Racloirs concaves & 2 \\
Racloir double convexe-concave & 1 \\
Grattoirs & 3 \\
Éclat tronqué & 1 \\
Retouches irrégulières & 12 \\
Divers : encoche/denticulé & 4 \\
Divers : encoche/grattoir & 2 \\
Divers : encoche/racloir & 6 \\
Divers : Denticulé/racloir & 1 \\
Divers : grattoir/racloir & 1 \\
Divers : outil sur galet & 2 \\
Nucléus & 101 \\
Couteaux à dos naturel & 3 \\
Éclats Levallois atypiques & 16 \\
Éclats et fragments d'éclats & 696 \\
non-retouchés & 1089 \\
Total & \\
\hline
\end{tabular}

Tabl. 3 - Indices réels et essentiels de la série H (Cagny-l'Épinette).

\begin{tabular}{|l|r|r|}
\hline \multicolumn{1}{|c|}{ Indices } & \multicolumn{1}{c|}{ réel } & ess. \\
\hline ILty & 5,47 & 0 \\
II & 5,47 & 5,79 \\
III & 1,36 & 1,44 \\
\hline IV & 13,01 & 13,76 \\
\hline IB & 3,63 & 3,83 \\
\hline IAu & 0 & 0 \\
\hline Encoches et denticulés & 71,91 & 76,08 \\
\hline IR & 5,47 & 5,79 \\
\hline
\end{tabular}

Tabl. 4 - Indices techniques de la série H (Cagny-l'Épinette).

\begin{tabular}{|l|r|}
\hline \multicolumn{2}{|c|}{ Indices techniques } \\
\hline IF & 12,5 \\
IFs & 3,28 \\
I Lam & 1,09 \\
\hline
\end{tabular}

entre 20 et $60 \mathrm{~mm}$ et enfin, $73 \%$ d'éclats dont l'épaisseur se situe entre 5 et $20 \mathrm{~mm}$, l'étude métrique révèle la présence d'éclats de taille réduite, et d'éclats plus longs que larges et fins. Les catégories de types de talons sont diversement représentées, les lisses et corticaux dominant les autres. Des talons facettés sont présents. Un certain nombre d'éclats ont des caractéristiques proches de celles d'éclats Levallois, ce qui a permis de les comptabiliser comme éclats Levallois atypiques. Leur caractère Levallois est possible en raison de la présence d'au moins un nucléus Levallois. Tous les éclats issus du test des blocs de matière première, de la mise en forme du nucléus, et du plein débitage sont présents et confortent l'impression d'un site où le débitage sur place semble avoir existé.

\section{- Les nucléus}

20 nucléus ont été recensés dans ce niveau. L'étude du degré d'exploitation des nucléus par surface de débitage rend compte des résultats suivants. Malgré une relative diversité des types de nucléus, dont certains comptent jusqu'à trois surfaces de débitage, les supports au débitage sont essentiellement à unique surface de débitage ( $80 \%)$. La productivité de ces surfaces de débitage est très variable d'un nucléus à l'autre. De façon générale, la gestion de ces surfaces est considérée comme "moyenne" puisque deux à trois enlèvements seulement ont été débités. De plus, les surfaces de débitage sont rarement exploitées dans leur totalité. Les cas les plus communs présentent un débitage qui n'intéresse que $50 \%$ de la surface de débitage.

L'étude générale des types de débitage observés par surface de débitage montre une grande homogénéité du débitage, organisé en type unipolaire ou bipolaire opposé. Ainsi, l'étude des nucléus à unique surface de débitage enregistre-t-elle une nette dominance de la méthode de débitage unipolaire $(81 \%)$, suivie quantitativement de loin par la bipolaire opposée. Quant à l'étude des nucléus à deux surfaces de débitage, elle rend compte des mêmes résultats que la catégorie précédente : le débitage unipolaire (66\%) et bipolaire opposé dominent ; associant parfois deux types différents sur un même nucléus.

II faut souligner la présence d'un nucléus Levallois à éclat préférentiel aménagé sur un rognon de silex assez plat dont la partie inférieure est entièrement corticale. La convexité distale de la surface de débitage a été obtenue par une série d'enlèvements. Par contre, il n'y a pas eu d'enlèvements latéraux de préparation, les convexités latérales résultant de la morphologie naturelle du rognon de silex (cf. Tuffreau et al., 1986 ; fig. $13, n^{\circ} 3$ ).

\section{- Données métriques}

$75 \%$ des nucléus ont une longueur supérieure à $60 \mathrm{~mm}$. Aucun n'entre dans les catégories supérieures à $140 \mathrm{~mm}$. En ce qui concerne les largeurs, les nucléus se ré- partissent uniformément entre les catégories de plus $(55 \%)$ et de moins (45\%) $80 \mathrm{~mm}$ de largeur. Enfin, $55 \%$ d'entre eux ont une épaisseur comprise entre 40 et $80 \mathrm{~mm}$. Ces nucléus sont donc dans l'ensemble assez massifs et souvent plus larges que longs.

\section{- L'outillage}

\section{- Le groupe biface et chopper/chop- ping-tools}

Il est insignifiant dans cette série. L'outillage est donc principalement constitué par les outils sur éclats qui représentent $25 \%$ des outils et des fragments d'éclats.

Le groupe biface est constitué de trois ébauches de bifaces, de quatre pièces entières, de deux pointes et de deux fragments. D'après les travaux de J. Léopold (1993), la forme prépondérante des bifaces est amygdaloïde, puis cordiforme. Les sommets sont en majorité pointus, puis arrondis et rectilignes. Les bases sont le plus souvent corticales, quant aux surfaces, elles ne sont pas toujours entièrement travaillées. Ces outils sont de petite dimension (longueur inférieure à $100 \mathrm{~mm}$ ) et peu épais.

\section{- Les outils classiques}

Ils représentent $94,2 \%$ des outils sur éclat et sont relativement diversifiés. La série est surtout composée par les encoches et denticulés. Si ces derniers restent les outils prépondérants, les racloirs (7\% des outils) ne sont pas à omettre dans cet ensemble (fig. 9). Ceux-ci, en effet, sont caractérisés par leur grande diversité typologique. Aucun racloir double n'a été recensé. Les racloirs simples sont donc les plus communs, le type dominant étant le racloir simple droit. En général, la retouche est rarement envahissante, le plus souvent courte et parallèle. Mis à part quelques grattoirs, il y a absence totale d'outils de type paléolithique supérieur. Un comportement spécifique a pu être observé dans le choix de la face à retoucher. De façon générale, les outils sont rarement alternes. On a souvent préféré la face supérieure aux autres. Les outils par catégorie, certes, présentent des variations dans ce choix. Les encoches sont essentiellement localisées sur la face supérieure alors que les denticulés ne le sont qu'exceptionnellement. Les racloirs et les grattoirs présentent plusieurs 
points communs : ils ne sont jamais alternes et sont réalisés sur les faces inférieures et supérieures du support.

\section{- Sélection des supports}

Dans $85 \%$ des cas, les éclats ont été choisis comme supports-outils contre $15 \%$ de fragments d'éclats. D'après le cortex restant sur les supports, on remarque que les éclats ont été choisis sans aucun tri spécifique. Divers types de fragments d'éclats ont été sélectionnés, mais le choix s'est davantage porté sur les fragments entièrement dépourvus de cortex. A l'intérieur des différentes catégories d'outils, on peut observer quelques variations de choix de supports selon l'outil considéré. Ainsi, le grattoir est réalisé dans $100 \%$ des cas sur éclat, les encoches et denticulés dans 85 à $88 \%$ des cas et enfin $75 \%$ pour les racloirs. Dans le détail, les racloirs et grattoirs sont presque toujours sur "éclats ordinaires", alors que les encoches et denticulés ont été réalisés à partir d'éclats divers. L'étude métrique de ces supports retouchés montre qu'en général, le choix s'est autant manifesté pour les supports de plus de $50 \mathrm{~mm} \mathrm{(42 \% )} \mathrm{que} \mathrm{de} \mathrm{moins} \mathrm{de}$ $50 \mathrm{~mm}$ (58 \%). Si les encoches confirment cette caractéristique, les denticulés et les racloirs présentent des résultats un peu plus nuancés. Pour ces deux catégories d'outils, le choix s'est surtout porté sur les éclats de plus de $50 \mathrm{~mm}$ car $71 \%$ des encoches et $75 \%$ des racloirs sont concernés par de tels supports.

\section{- Les outils composites}

Les outils composites représentent $5,8 \%$ de l'outillage sur éclat et s'expriment par une grande variété d'associations, même si ces dernières sont quantitativement faiblement représentées. Des six associations principales, seule celle du "denticulé-grattoir" est absente. Un comportement spécifique entre les outils a pu être remarqué. De façon générale, aucun outil composite n'est fait de façon alterne. La localisation des deux groupes d'outils sur la même face et notamment sur la face supérieure $(92,8)$ est quasi-exclusive dans le niveau $\mathrm{H} .73 \%$ des supports sélectionnés ont une dimension supérieure à $50 \mathrm{~mm}$ contre $42 \%$ dans les outils classiques.

\section{- Diagnostic}

Le niveau $\mathrm{H}$, le plus récent de la séquence fluviatile du gisement de Cagny-l'Épinette se caractérise par de rares bifaces, de rares choppers et par un outillage sur éclat classique et composite diversifié. Ce niveau est sans débitage Levallois et sans hachereaux. Malgré un certain nombre de nucléus à multiples surfaces de débitage, les plus fréquents sont les nucléus à une seule surface de débitage avec un débitage unipolaire. Malgré tout, la productivité de ce type de débitage et des surfaces est assez faible. Les outils sur éclats sont dominés par les outils classiques surtout composés d'encoches, de denticulés et de racloirs. Ces derniers sont typologiquement très variés et participent pleinement à la composition des outils composites aux côtés des encoches. Les racloirs simples droits sont les plus communs. Ces outils sont réalisés sur éclats plus ou moins corticaux et sur éclat ordinaire sans aucune spécificité technologique. En dehors des grattoirs, les outils de type paléolithique supérieur sont inexistants. Trois chaînes opératoires ont pu être reconnues dans cet assemblage : l'une à production de quelques bifaces, l'une à production de quelques choppers et une dernière à production d'éclats à partir de nucléus non Levallois.

Compte tenu de la rareté des bifaces, par rapport aux choppers, et de la bonne représentativité d'un outillage sur éclats diversifié (outils composites inclus), cette série semble se différencier nettement des autres assemblages acheuléens, tant à Cagny-l'Épinette que dans d'autres gisements de la région. II est difficile d'apprécier si cette originalité résulte de l'échantillonnage en raison d'activités spécialisées ou d'autres facteurs.

\section{Analyse préliminaire de la série lithique de I1}

La série lithique des niveaux 11 et $11 \mathrm{~b}$, qui a été étudiée (campagne de fouille 1993 incluse), comprend 420 artefacts ainsi qu'un certain
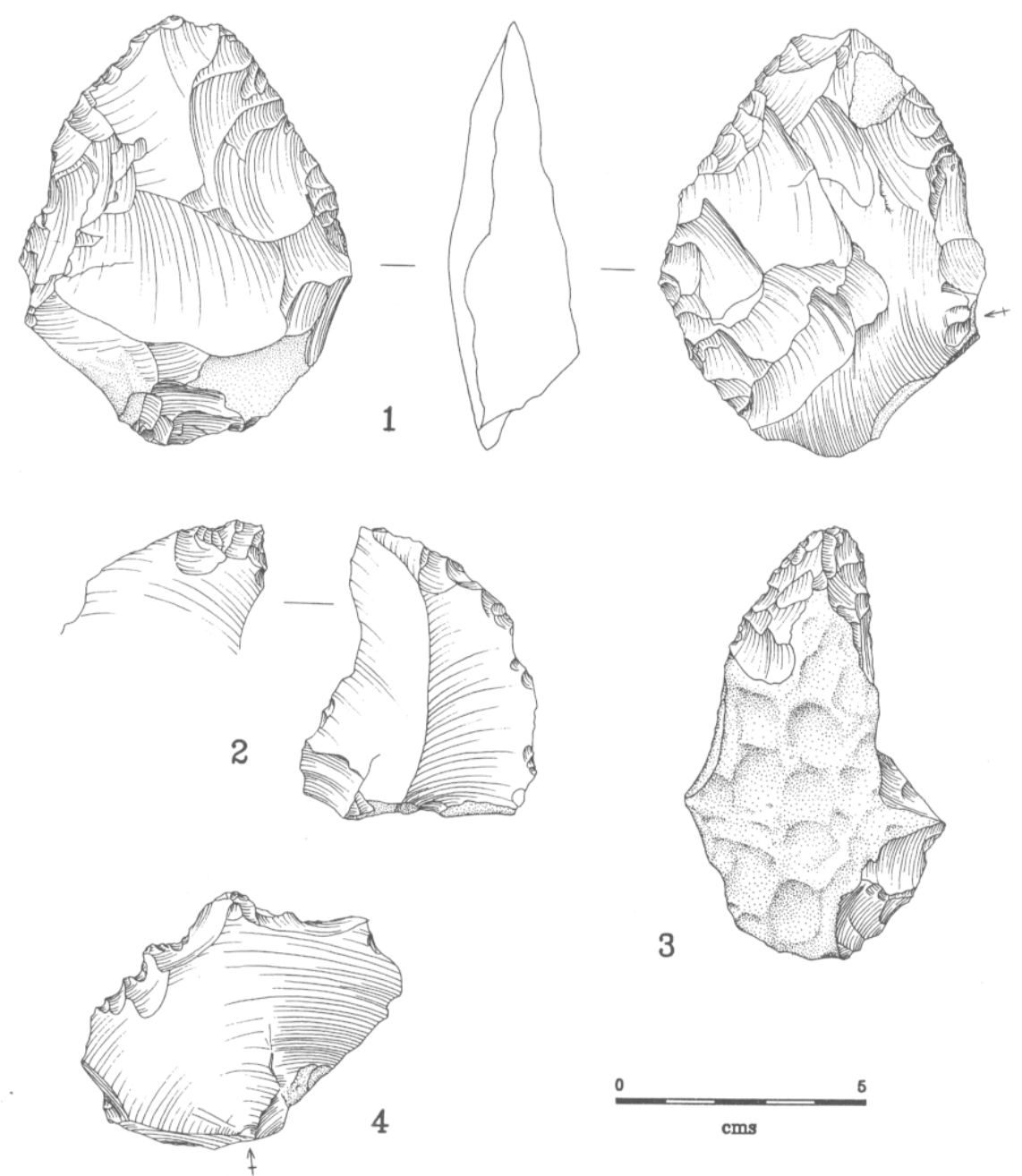

Fig. 10 - Cagny-l'Épinette, niveau 11, industrie lithique. 1 : biface sur éclat ; 2 : éclat à retouches irrégulières avec amincissement à la partie distale ; 3 : éclat de gel avec des retouches distales ; 4 : denticulé inverse. 
nombre de pièces en silex non travaillées (gélifracts et de rognons de silex). La série comprend notamment un grand nombre d'éclats non retouchés (194 pièces complètes ou parties proximales), 49 outils (complets ou parties proximales), 8 fragments d'outils et 24 bifaces, le reste se composant de fragments d'éclats et de débris (fig. 10 et 11). II y a aussi 30 nucléus, la plupart d'entre eux n'ayant été que peu exploité et montrant pour beaucoup d'entre eux que la trace d'un ou de deux enlèvements (tabl. 5).

Sur le plan technologique, la série est plutôt indéfinissable. II y a très peu d'éclats de taille de bifaces reconnaissables et pratiquement pas de produits Levallois. L'absence d'éclats de taille de bifaces semble indiquer qu'il n'y a pas eu, lors de l'occupation, de fabrication de bifaces sur place, ce qui est confirmé par la présence d'un biface fait dans une qualité de silex qui n'est pas présente sur le site. La plupart des talons sont lisses ou corticaux $(N=177)$. Les facettes sont au nombre de 15 et les dièdres au nombre de 5 (IF = 10,2; IFs = 7,6). Aucune lame n'est présente. La série est fortement corticale. Un quart seulement des éclats ne présentent pas de cortex ce qui indique que les nucléus ont été peu exploités bien cléus est plutôt élevé $(8,1$ proximaux ou entiers). que le nombre de produits par nu-

Tabl. 5 - Caractéristiques et indices typologiques de I1 et I1b.

\begin{tabular}{|c|c|c|c|}
\hline Type & Nombre & \% réel & $\%$ ess. \\
\hline 2. Éclat Levallois atypique & 1 & 0,44 & - \\
\hline 9. Racloir simple droit & 10 & 4,42 & 8,55 \\
\hline 10. Racloir simple convexe & 7 & 3,10 & 5,98 \\
\hline 15. Racloir double convexe & 1 & 0,44 & 0,85 \\
\hline 21. Racloir déjeté & 1 & 0,44 & 0,85 \\
\hline 23. Racloir transversal convexe & 1 & 0,44 & 0,85 \\
\hline 25. Racloir sur face plane & 2 & 0,88 & 1,71 \\
\hline 26. Racloir à retouche abrupte & 1 & 0,44 & 0,85 \\
\hline 29. Racloir à retouches alternes & 3 & 1,33 & 2,56 \\
\hline 30. Grattoir typique & 2 & 0,88 & 1,71 \\
\hline 31. Grattoir atypique & 1 & 0,44 & 0,85 \\
\hline 32. Burin typique & 2 & 0,88 & 1,71 \\
\hline 33. Burin atypique & 1 & 0,44 & 0,85 \\
\hline 34. Perçoir typique & 1 & 0,44 & 0,85 \\
\hline 36. Couteau à dos typique & 1 & 0,44 & 0,85 \\
\hline 37. Couteau à dos atypique & 1 & 0,44 & 0,85 \\
\hline 38. Couteau à dos naturel & 16 & 7,08 & - \\
\hline 39. Raclette & 4 & 1,77 & 3,42 \\
\hline 40. Éclat tronqué & 2 & 0,88 & 1,71 \\
\hline 42. Encoche & 39 & 17,26 & 33,33 \\
\hline 43. Denticulé & 25 & 11,06 & 21,37 \\
\hline 45. Retouches sur face plane & 2 & 0,88 & - \\
\hline 48. Retouches abruptes alternes épaisses & 85 & 37,61 & - \\
\hline 50. Retouches bifaciales & 5 & 2,21 & - \\
\hline 54. Encoche en bout & 1 & 0,44 & 0,85 \\
\hline 59. Chopper & 1 & 0,44 & 0,85 \\
\hline 61. Chopping-tools & 1 & 0,44 & 0,85 \\
\hline 62. Divers & 9 & 3,98 & 7,69 \\
\hline Total réel & & & 226 \\
\hline Total essentiel & & & 117 \\
\hline Éclats complets et fragments proximaux & & & \\
\hline $\begin{array}{l}\text { (y compris pieces tecnnologiques) } \\
\text { Fragments et esquilles }\end{array}$ & & & 133 \\
\hline Nucléus et fragments & & & 30 \\
\hline Bifaces & & & 24 \\
\hline
\end{tabular}

La composante retouchée est également indéfinissable. II y a 226 pièces en décompte réel mais près d'une centaine d'entre elles sont des pièces à retouche alterne

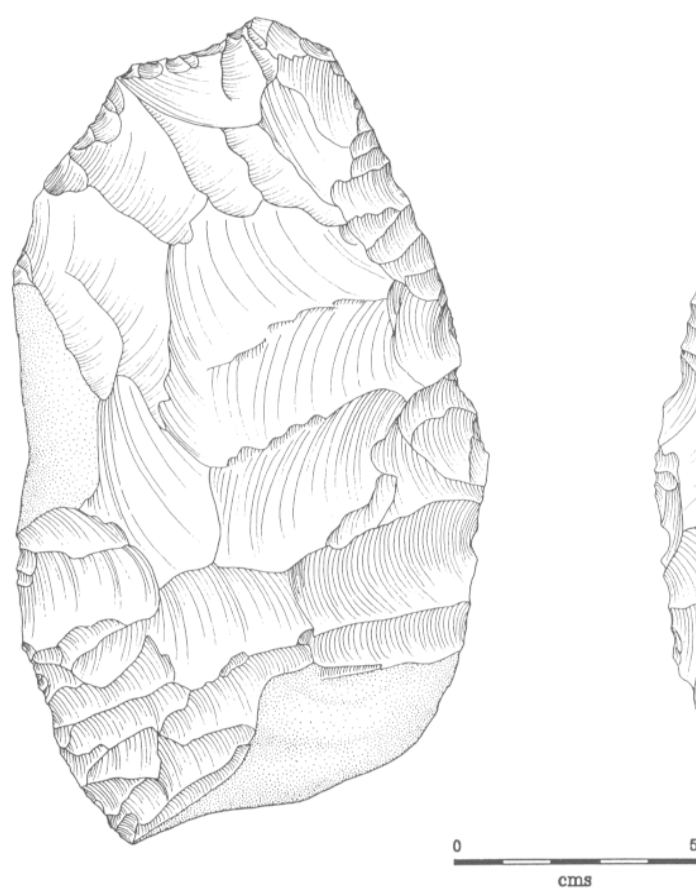

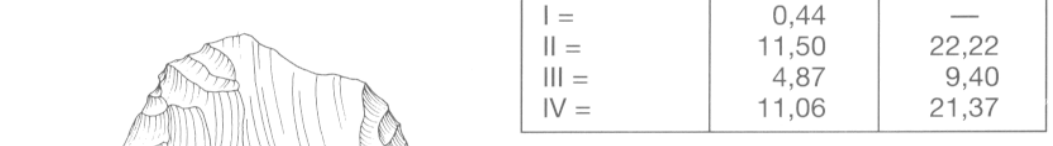

abrupte (types 46-47). Le décompte essentiel inclut quelques racloirs, surtout des simples et une proportion beaucoup plus forte d'encoches et de denticulés. II y aussi quelques outils de type paléolithique supérieur, des burins, des grattoirs, des couteaux à dos et deux pièces tronquées à retouches inverses. A l'exception des bifaces, les outils sont assez grossiers et non standardisés. II y a une forte proportion d'outils retouchés aménagés sur des gelifacts, notamment un biface partiel, ce qui limite beaucoup les possibilités d'analyses des chaînes opératoires.

Les bifaces du niveau 11 sont conformes au caractère général de l'industrie lithique du fait de la variabilité de leur morphologie (en majo-

Fig. 11 - Cagny-l'Épinette, niveau I1, industrie lithique. Grand biface en silex allochtone. 
rité pointues, mais avec des types allant de la limande et du cordiforme au lancéolé), leur dimension (80 à 160 $\mathrm{mm}$ de longueur) et de leur technologie (rognons de silex disponibles sur place, à l'exception de la pièce déjà mentionnée, un gelifact et un éclat). La forte variabilité des bifaces pourrait suggérer un mélange de multiples occupations, à moins que la dimension, la morphologie et la technologie aient moins d'importance que la réalisation de l'arête du biface.

L'absence de standardisation des outils amène à se demander s'il s'agit d'une conséquence de l'âge de l'industrie, d'une utilisation très faible (cf. Dibble et Rolland, 1992) ou de phénomènes sédimentaires comme semblerait le suggérer l'état des tranchants de nombreuses pièces.

\section{- LA GRANDE FAUNE}

L'étude des restes osseux de Cagny-l'Épinette réalisée par A.-M. Moigne entre 1984 et 1994 a permis de décrire les os sous leur aspect paléontologique, leur aspect taphonomique, d'après l'inventaire et les traces observées, puis de développer l'étude des populations de grands herbivores pour les niveaux archéologiques les plus riches. Cette étude est complétée par une analyse des traces d'origine anthropique pour mieux comprendre la fonction de ce gisement et les différences observées entre les niveaux d'occupation.

L'étude paléontologique des restes osseux permet de décrire les différents genres regroupés sur le site et d'évaluer leur degré évolutif par rapport aux gisements contemporains. Cette analyse s'effectue d'après les critères morphologiques et biométriques. Pour ces derniers, il est d'usage d'employer des abréviations telles que Diamètre transversal $=\mathrm{Dt}$; Diamètre antéro-postérieur $=$ Dap, Longueur $=\mathrm{L}$, largeur $=\mathrm{I}$, Hauteur $=H$. Nous avons ajouté à ces mesures classiques la hauteur de la diaphyse des os dont l'épiphyse n'est pas soudée, hauteur $=h$. Les dimensions sont données en millimètres.

\section{Les espèces de grands mammifères}

\section{- VULPES SP.}

Une branche horizontale de mandibule sans dent a été découverte dans le niveau I, mais les traces ob-

Fig. 12 - Cagny-l'Épinette, niveau 11. Squelette éclaté d'aurochs (d'après Helmer, 1987) montrant, à gauche, fracturation volontaire et encoches de percussion, à droite, stries de décarnisation et de désarticulation et zones de piquetage (xxx).

servées sur les épiphyses d'os long dans les autres niveaux peuvent également être attribuées à un petit carnivore de la taille du renard.

\section{- Crocuta sp.}

La hyène est présente dans le niveau I ou a été découvert une extrémité distale d'humérus. L'épicondyle est rongé et interdit toute mesure. Sa taille correspond à un humérus de hyène tachetée actuelle.

\section{- Cervus elaphus}

Les 276 restes attribués aux grands cervidés sont présents dans chacun des niveaux fouillés.
Bien que le niveau 11 soit le plus riche, le cerf est dominant dans le niveau I.

La forme des bois de Cerf de Cagny-l'Épinette n'est pas complètement connue. Comme sur la plupart des sites préhistoriques, les bois sont fragmentés. La base du merrain comprenant les deux andouillers est conservée sur plusieurs bois de chute. Le premier andouiller est nettement plus fort que le second et fortement recourbé à son extrémité. L'ornementation n'est pas très développée sauf sur quelques andouillers. Un épois écrasé présente au moins trois pointes. 
Fig. 13 - Cagny-l'Épinette, niveau I1. Squelette éclaté de cerf (d'après Helmer, 1987) montrant à gauche, fracturation volontaire et encoches de percussion, à droite, stries de décarnisation et de désarticulation et zones de piquetage (xxx).
Dimensions de la base pour le spécimen le plus gros :

Ép. $86,18 A, I J, 860: 62 \times 52$ et 185 de circonférence.

Les dents de cerf sont plutôt rares et en mauvais état de conservation dans les niveaux $\mathrm{H}$ et $\mathrm{I}$, toutefois leurs mesures sont homogènes dans les niveaux I, I1, IJ et $\mathrm{J}$. Ces mesures sont faibles par rapport au corpus de Cerfs du Pléistocène moyen (tabl. 6).

La taille des $\mathrm{m}^{3}$ inférieures se rapproche de celle de Orgnac 3 (Aouraghe, 1992) et de Hoxne en Angleterre (Stuart et al., 1993). Les dents découvertes à Clacton et à Swans-
Châtillon-Saint-Jean, Vaufrey, Lazaret etc...).

Les dents supérieures sont également petites. On peut considérer que la population de Cagny-l'Épinette est homogène par rapport à des populations contemporaines (tabl. 7).

Certains auteurs ont considéré l'augmentation de la taille des molaires de cervidés du stade 9 au stade 6 , comme un critère chronologique (Serre, 1993). On peut donc considérer ce site comme relativement ancien dans cette phase. II est intéressant de noter que le même phénomène se reproduit dans la phase de refroidissement du Mindel (stades 17 à 14), et lors de la dernière glaciation (stades 5 à 2). En effet, les cervidés du Würm ancien ont pu être individualisés par divers caractères et surtout leur petite taille en Cervus elaphus simplicidens Guadelli. Pour cet auteur, ce petit cerf est toutefois contemporain d'un cerf plus grand (Guadelli, 1987).

\section{- Le squelette post-céphalique}

Seul le radius peut être comparé à ceux des autres sites. Les dimensions sont également faibles, de l'ordre de grandeur des radius du cerf élaphe de Hoxne et d'Orgnac 3. Les os de La Fage et de Tourville La Rivière sont plus robustes. Les os longs des sites plus récents tels que Châtillon-Saint-Jean, Lazaret, Vaufrey, Fontéchevade et l'Observatoire sont nettement plus imposants. On peut noter que l'espèce de petite taille décrite au Würm ancien présente des dimensions comparables.

\section{- DAMA SP.}

Les ossements de daims comprenant trois métacarpiens une phalange et deux fémurs sont caractéristiques mais fracturés. Les bois sont des fragments de palmure qui ne Toutes les dents des gisements français sont plus robustes (La Fage,
Tabl. 6 - Mensurations des dents de cerfs du Pléistocène moyen.

\begin{tabular}{|c|c|c|c|c|}
\hline & & Cagny-l'Épinette & Hoxne & Orgnac 3 \\
\hline D4 & $\mathrm{L}_{1}^{\mathrm{n}}$ & $\begin{array}{c}1 \\
27,7 \\
10,4\end{array}$ & & \\
\hline M1 & $L_{1}^{n}$ & $\begin{array}{c}2 \\
20-22 \\
13,3-13,9\end{array}$ & & $\begin{array}{l}23 \\
23 \\
14,2\end{array}$ \\
\hline M2 & $\mathrm{L}_{1}^{\mathrm{n}}$ & $\begin{array}{c}2 \\
22,2 \\
14,1-14,9\end{array}$ & $\begin{array}{r}1 \\
21 \\
15\end{array}$ & $\begin{array}{l}20 \\
24,7 \\
15,5\end{array}$ \\
\hline M3 & $L_{1}^{n}$ & $\begin{array}{c}2 \\
30 \\
14\end{array}$ & $\begin{array}{c}1 \\
29,5 \\
15,1\end{array}$ & $\begin{array}{l}19 \\
31,5 \\
14,3\end{array}$ \\
\hline
\end{tabular}


permettent pas de connaître la forme dans son ensemble. II est dans ce cas difficile de l'attribuer à Dama clactoniana, bien qu'il soit ainsi nommé dans les gisements contemporains comme Atapuerca ou Orgnac 3. La dent, une deuxième déciduale inférieure $(L=10,4 ; I=5)$ semble indiquer un daim de taille assez forte comme celui décrit au Pléistocène moyen.

\section{- Bos PRIMIGeNIUS}

Le matériel attribué à cette espèce est important et provient de chaque niveau, mais 11 est le plus riche. Le nombre d'individus n'est pas très élevé comparé au nombre de restes car il semble que les squelettes de veaux, torillons et génisses soient pratiquement entiers. Les chevilles osseuses de jeunes mâles font $94,5 \mathrm{~mm}$ de diamètre, ces chevilles osseuses sont très fortes pour des animaux de moins de 3 ans.

Sur la dernière molaire inférieure, le dernier lobe est situé dans l'alignement antéro-postérieur des deux autres lobes, ce qui caractérise le genre Bos. La forme du sillon entre les deux lobes postérieurs est ouverte et non fermée comme chez le bison. Les dimensions de cette dent sont comparables à celles d'Orgnac 3.

La plupart des éléments du squelette post-céphalique sont fragmentaires. Lorsqu'il a été possible de faire la même mesure sur des os de niveaux différents, elle est homogène. Pour le membre antérieur, seul le radius a été mesuré. Le relief latéral d'insertion est très développé sur l'épiphyse proximale. L'apophyse de l'ulna est profonde et bien dessinée. Ces critères morphologiques indiquent le genre Bos. La largeur articulaire proximale $(D T=102$ ) sort des limites de variation de l'aurochs femelle de Lunel-Viel et correspond davantage à celui des mâles de cette population (Brugal, 1984).

Le métacarpe entier (11) est assez grand mais pas très robuste. II peut s'agir d'un jeune individu de 4 ans dont les insertions ne sont pas très marquées ou d'une femelle (tabl. 9).

Pour le membre postérieur, l'extrémité distale du tibia montre la même tendance (Brugal $7=58$; Brugal $9=50$ ). Les dimensions de talus entiers rentrent bien dans les limites de variation de la population femelle de Lunel-Viel $(\mathrm{Ht}=88$; $\mathrm{Dt}=6,3$ et
Tabl. 7 - Dents supérieures de cervidés du Pléistocène moyen.

\begin{tabular}{|l|c|c|c|}
\hline & Cagny-l'Épinette & Orgnac 3 & Hoxne \\
\hline Diamètre antéro-postérieur & 22 & 24,1 & 21,5 \\
Diamètre mésio-distal & 23 & 25 & 24,9 \\
\hline
\end{tabular}

Tabl. 8 - Mesures des dents d'aurochs (OS d'adultes, I1, IJ et J).

\begin{tabular}{|c|c|c|c|c|c|c|}
\hline \multicolumn{5}{|c|}{ Dents supérieures } & \multicolumn{2}{|c|}{ Dents inférieures } \\
\hline & & $\mathrm{n}$ & $\mathrm{m}$ & $\mathrm{H}$ & $\mathrm{n}$ & $\mathrm{m}$ \\
\hline D2 & $\begin{array}{l}\mathrm{e} \\
\mathrm{L}\end{array}$ & & & & $\begin{array}{l}3 \\
3\end{array}$ & $\begin{array}{c}8,03 \\
10,7\end{array}$ \\
\hline \multirow[t]{2}{*}{ D3 } & $\mathrm{e}$ & 3 & 19,15 & & 4 & 11,6 \\
\hline & $\mathrm{L}$ & 2 & 25,6 & & 4 & 20,9 \\
\hline \multirow[t]{2}{*}{ D4 } & $\mathrm{e}$ & 6 & 23,43 & & 5 & 16 \\
\hline & $\mathrm{L}$ & 6 & 26,2 & & 6 & 37 \\
\hline \multirow[t]{2}{*}{ M1 } & $\mathrm{e}$ & 7 & 24,1 & & 11 & 16,5 \\
\hline & $\mathrm{L}$ & 7 & 33 & & 11 & 34,5 \\
\hline \multirow[t]{2}{*}{ M2 } & e & 10 & 26,6 & 58 & 8 & 18 \\
\hline & $\mathrm{L}$ & 10 & 31,3 & & 8 & 36,4 \\
\hline \multirow[t]{2}{*}{ M3 } & $\mathrm{e}$ & 3 & 27,5 & & 4 & 18,7 \\
\hline & L & 3 & 36,1 & & 4 & 44,2 \\
\hline \multirow{2}{*}{ P2 } & $\mathrm{e}$ & 4 & 15,3 & 37 & & \\
\hline & $\mathrm{L}$ & 4 & 21,2 & & & \\
\hline \multirow[t]{2}{*}{ P3 } & $\mathrm{e}$ & 5 & 176,6 & & 1 & 15,6 \\
\hline & $\mathrm{L}$ & 5 & 21 & & 1 & 24,3 \\
\hline \multirow[t]{2}{*}{ P4 } & $\mathrm{e}$ & 2 & 21 & 39,8 & 1 & 14,2 \\
\hline & L & 3 & 24 & & 1 & 25 \\
\hline
\end{tabular}

Tabl. 9 - Dimensions des métacarpes de Bos.

\begin{tabular}{|l|c|c|c|}
\hline & Épinette & Tourville & Orgnac 3 \\
\hline H & 256 & $250-258$ & $249,265,269$ \\
Dtp & 73 & & \\
dapp & 45 & & \\
Dtm & 45 & $41-44,5$ & $43-58$ \\
dapm & 32 & $30-34$ & $31,5-43$ \\
\hline
\end{tabular}

Dap $=52$ ) ainsi que celles des cubonaviculaires $(\mathrm{Ht}=65 ; \mathrm{Dt}=71$ et Dap = 70) (tabl. 10).

Les deux métatarses aduites complets de Cagny-l'Épinette sont très différents. Le métatarse (Ep 90, $20 \mathrm{Q}, 11,25)$, le plus grand et trapu peut correspondre à la population mâle. Le deuxième est beaucoup plus gracile et diffère morphologiquement.
Pour les jeunes animaux, les phalanges antérieures et postérieures ont pu être séparées mais les phalanges adultes sont trop rares (tabl. 11).

Ces dimensions sont comparables à celles d'Orgnac 3 et nettement plus faibles que celles de Tourville-la-Rivière (Descombes, 1983).

En conclusion, les os de bovidés de Cagny-l'Épinette peuvent tous être attribués au genre Bos. Comme pour les populations du Pléistocène moyen, les os présentent un fort dimorphisme sexuel. Leur grande taille les rapproche de la population de Lunel-Viel considérée comme une population de référence, attribuée à Bos primigenius trochoceros.
Tabl. 10 - Mensurations de métatarses de Bos.

\begin{tabular}{|c|l|l|l|l|l|}
\hline & Épinette & \multicolumn{1}{|c|}{ Lunel-Viel f } & Lunel-Viel m & Orgnac 3 & Tourville \\
\hline Ht & $300 \quad 280$ & $277,9-312,17$ & $263,7-300$ & & \\
DTp & $75,4 \quad 60,5$ & $65,8-78,7$ & $53,4-67,3$ & $62,2-68,5$ & $59-71$ \\
dap & $75,1-57$ & $61,2-74,6$ & $54,8-66,2$ & $62,2-68,5$ & $58-71$ \\
Dtm & 49 & 39 & $45-55$ & $35,7-45,8$ & \\
dap & 47 & 38 & $43,8-52,7$ & $38,3-45,4$ & \\
\hline
\end{tabular}

Tabl. 11 - Mensurations des phalanges de Bos (11).

\begin{tabular}{|c|c|c|}
\hline & Première phalange : & Deuxième phalange : \\
\hline Ht & $74,5-73,5$ & 49 \\
Dtp & $34-41,5$ & 33 \\
dap & 32,5 & 38 \\
Dtd & 32,5 & 30,5 \\
Dap & 27,7 & 32 \\
\hline
\end{tabular}




\section{- EquUs caballus mOSBachensis}

Les chevaux sont présents dans tous les sols. Les dents supérieures rapportées aux niveaux $\mathrm{F} 2$ et $\mathrm{H}$ sont particulièrement petites (moyenne des longueurs $(L)$ des 3 prémolaires : 29,4 ; moyenne des longueurs des protocônes (Lp : 13,3). L'indice protoconique est de 46 . Les dents supérieures provenant des niveaux 11 et $J$ sont nettement plus robustes (prémolaire : $L=32$; $L p=16$; molaire : $L=30,5 ; L p=14,4$, dent non usée). La P2 inférieure gauche du niveau I correspond à ce type de cheval.

Le métatarse entier de Cagnyl'Épinette provient du niveau 11, il est particulièrement long ( $L=295$ ) et correspond aux plus grands chevaux anté-wurmiens. L'extrémité distale est large ( $d t=57,5)$, la largeur susarticulaire est inférieure à cette mesure.

Les os du carpe, du tarse et l'extrémité distale d'humérus (DT articulaire distal $=86 ;$ dap distal $=90,5$ ) sont également des os de bonne taille. Le diamètre de la diaphyse de l'humérus est faible, ce qui correspond à un os gracile.

D'après V. Eisenmann (1991), les dimensions et les proportions des dents ainsi que la largeur distale des métapodes correspondent à différents types. La ressemblance avec E. achenheimensis (L.A.I.) est notable (Wernert, 1957). Ce dernier, ainsi que le cheval de Hoxne, présente une largeur articulaire supérieure. La caractéristique des chevaux de Cagnyl'Épinette est leur grande taille. Ils apparaissent toutefois comme les plus graciles des $E$. mosbachensis (Prat, 1968).

Les chevaux des niveaux supérieurs ne sont malheureusement connus, de par les conditions de conservation, que par des dents qui sont de très petite taille.

\section{- EQUUS HYDRUNTINUS}

Sa présence sur le site n'est attestée que dans le niveau $\mathrm{H}$, par une phalange ainsi qu'une diaphyse de fémur adulte de très petite taille pour le cheval. Sa présence à Cagnyl'Épinette n'est pas surprenante car l'âne sauvage est connu sur plusieurs sites contemporains.

\section{- Elephas (Palaeloxodon) antiquus}

L'éléphant a été déterminé dans le niveau $H$. II s'agit de deux lames contiguës $(I=40 ; \mathrm{Ht}=50)$ écartées

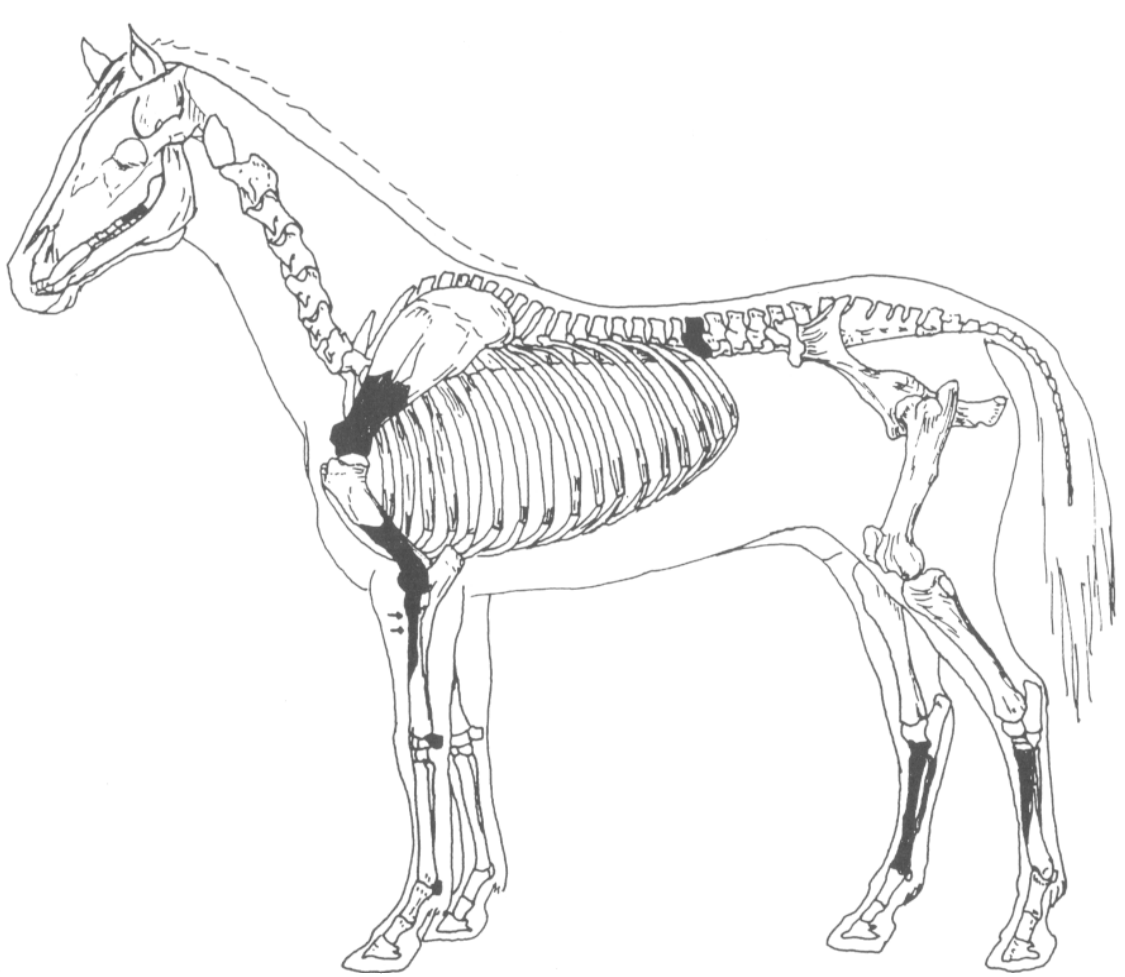

Fig. 14 - Cagny-l'Épinette, niveau I1. Ossements de cheval présents avec localisation des encoches de percussion et des plages de piquetage.

de $10 \mathrm{~mm}$. II s'agit d'une D4 inférieure. L'émail est fortement ridulée et épaisse. La base de la lame montre un pli au niveau du sinus médian, c'est pourquoi nous l'attribuons à l'éléphant antique.

\section{- Inventaire des restes osseux}

L'inventaire est étudié couche par couche mais nous ne développons ici que la conservation des ossements dans les différents niveaux qui comprennent une quantité d'ossements suffisant pour l'analyse taphonomiques. La répartition spatiale ne sera pas abordée.

\section{- Niveau H}

Les os longs de bovidés ainsi que les dents représentent chacun $25 \%$ du matériel conservé. Les dents sont isolées et le plus souvent fragmentées et altérées. Une cheville osseuse presqu'entière est conservée. Les côtes et les vertèbres sont fragmentées. Les os longs, cassés, conservés sous forme de grosses esquilles, proviennent d'individus adultes. Les traces observées sur les ossements sont des fissures dues à diverses causes comme le gel et l'eau. Ces facteurs édaphiques ont favorisé la disparition des os de petite taille et altéré les dents. Les es- quilles présentent de nombreuses traces de charriage. Le cubo-naviculaire est altéré par les racines.

Les ossements de cervidés sont dans le même état de conservation. Les dents sont isolées, le plus souvent fragmentées. Les os courts sont rares et altérés, seul le rocher a subsisté parmi les os du crâne. Les os longs sont peu nombreux. Une des esquilles a été rongée par des petits carnivores.

Le cheval est surtout représenté par les dents supérieures. Les os longs sont fragmentés.

En conclusion, la conservation dans le niveau $H$ est mauvaise. Seules les dents et les éléments les plus gros ont résisté à l'altération du sol (moyenne des os pour ce niveau : $57 \mathrm{~mm}$ ). La conservation différentielle des niveaux tels que $G, G X$ et $\mathrm{H} 1$ et $\mathrm{Hx}$ est tout à fait comparable.

\section{- Niveau I}

La conservation des ossements de bovidés de ce niveau donne un fort pourcentage des dents et des os longs : $50 \%$ des restes. Les dents supérieures sont regroupées sur les maxillaires. Les fémurs et les humérus sont absents de ce niveau. Les métapodes sont représentés par des condyles de jeunes non soudés. Les ossements d'adultes, radius, ulna, 
tarse, phalange et métapode sont fracturés.

Les os de cervidés sont également très morcelés. Parmi les fragments de bois, relativement peu nombreux, un bois de massacre a été conservé. Les dents sont isolées et les os longs, particulièrement les métapodes, sont fréquents. II y a encore un net déficit en os courts.

Les os d'équidés sont rares : deux os du carpe, un fragment de métacarpe et une dent isolée.

Les traces observées correspondent à des zones altérées par les racines. Bien que le niveau I recèle un humérus de hyène, il n'a pas été possible de mettre en évidence des traces de son activité sur les os.

\section{- NIVEau /1}

Pour l'aurochs, les dents ne représentent que $9 \%$ du matériel. Les chevilles osseuses sont trouvées en connexion avec les crânes mais ces derniers sont à l'état de fragments. Les vertèbres comptabilisées ici sont assez complètes et très souvent en connexions de trois ou quatre pièces.

Les os courts sont entiers, souvent en connexion anatomique. Les phalanges adultes sont fracturées.

Les os longs adultes sont également systématiquement fracturés, sauf certains métapodes, et portent des traces d'origine anthropique. Les os longs de jeunes bovidés sont entiers et il est souvent possible de regrouper les épiphyses et les diaphyses du même os et parfois de les apparier. Les connexions carpe-métacarpe et tarse-métatarse-phalanges ainsi que tibia-tarse sont hautement probables et permettent de bien décrire les individus regroupés dans ce niveau. La conservation est excellente. Seules les épiphyses de jeunes présentent quelques traces d'un carnivore, probablement le renard, dont on a retrouvé une mandibule. Les os portent de rares traces de charriage, fines et superficielles. La mandibule d'adulte âgé présente également une face altérée par les racines.

Les fragments de bois de cervidés sont très fréquents, soit plus d'un tiers du matériel. Ils correspondent à de nombreux andouillers et pointes d'andouillers. Trois associations regroupant les andouillers basilaires et des tronçons de merrain peuvent être attribuées aux bois de chute. Les dents isolées sont rares et une mandibule de jeune cerf provient de ce niveau : les os courts (carpien, tarsien, sésamoïdes et phalanges) sont peu nombreux mais présents. Tous les os longs sont représentés, fracturés, les métapodes étant les plus nombreux.

Certains ossements d'équidés ont pu être regroupés : naviculaire, cunéiforme, métatarse III et IV, en une portion de patte arrière droite, les autres ossements sont fracturés, $y$ compris les dents.

Tout semble indiquer que ce niveau a été très rapidement enfoui, ce qui a favorisé une excellente conservation et un remaniement très faible. L'extrême fragmentation des os comme les côtes, les vertèbres et les crânes de jeunes semble résulter d'un écrasement secondaire.

\section{- Niveau ls}

Si les restes de bovidés sont très bien conservés dans ce niveau, il semble qu'il y ait une sur représentation des dents et des os longs par rapport à la couche 11 par exemple. Les os courts sont relativement rares, représentés par des phalanges jeunes et adultes. Les os longs sont entiers sans épiphyses. Les condyles de métapodes sont nombreux.

Le niveau IJ n'a pas livré de traces particulières. Les diaphyses de jeunes bovidés sont écrasées en place ainsi que le crâne. Les vertèbres et les côtes sont entières, parfois écrasées mais en connexion par tronçons. L'absence relative des os du carpe semble indiquer que ce niveau se continue hors de l'espace fouillé.

Les os de cerf (20) sont surtout des fragments de bois regroupés en deux bois de chute dont l'un est assez imposant. Les os longs sont bien représentés, il n'y a ni os courts ni vertèbres. Bien que les dents et les os courts soient rares sur ce niveau, il ne semble pas qu'il y ait eu un transport du matériel car les cartilages des os longs de jeunes bovidés se sont défaits sur place, les bois sont écrasés sans transport post-dépositionnel. II semble simplement que l'ensemble du matériel de cette occupation n'est pas encore découvert.

\section{- Niveau J}

Les restes d'aurochs représentent la moitié des restes du niveau J. Les proportions générales se rapprochent de celles du niveau I, les dents sont nombreuses, elles sont le plus souvent isolées. Les fragments de crânes sont de fortes tailles, bien déterminables, les chevilles sont séparées. La plupart des vertèbres sont dispersées. Les côtes sont au contraire regroupées par 2 ou 3 , mais rarement entières.

Les os du tarse ont pu être assortis mais ne se trouvaient pas en connexion sur le site. Les scapulas sont bien représentées, l'une des jeunes est entière. Les os longs, humérus et fémurs sont entiers. Sur l'un des humérus, il a été possible d'associer la tête articulaire non soudée. Les radius sont mieux représentés par les adultes, pour l'individu jeune, le radius peut être associé à l'ulna. Les tibias sont regroupés, diaphyses et épiphyses non soudées et appariées. Les ossements d'aurochs sont bien conservés, toutes les parties squelettiques sont présentes pour les individus jeunes mais les connexions sont nettement plus lâches que dans les niveaux IJ et 11 . II est possible d'attribuer cette dispersion au passage, les esquilles sont de tailles réduites et portent parfois des stries de charriage. Le radius adulte présente des traces de carnivores et le jeune, des empreintes de dents de rongeurs. Les ossements de cette couche sont parfois altérés, ils peuvent avoir subi une exposition à l'air plus importante que pour les niveaux IJ et I1 par exemple.

Les fragments de bois de cervidé sont nombreux dans ce niveau et correspondent au moins à trois bois de chute et un bois de massacre, les restes crâniens proviennent au moins de deux crânes. Les côtes et vertèbres sont présentes dans ce niveau et les dents sont plus nombreuses. Les os longs sont bien représentés, portant des traces de carnivores, les métapodes et les phalanges sont absents. Les ossements sont très dispersés sur toute la superficie de la partie fouillée de J.

Les ossements d'équidés correspondent à des dents isolées et deux métapodes.

\section{- Étude de la population d'herbivores, pour les différents niveaux archéologiques}

Le problème de la détermination de l'âge et du sexe des animaux regroupés sur les différents niveaux du gisement ne peut malheureusement pas encore se poser en termes sta- 
tistiques mais la population de bovidés par exemple comprend une vingtaine d'associations dentaires dont les deux tiers sont jeunes, avec des dents lactéales. Cette proportion importante des jeunes individus peut s'observer sur les dents, sur la taille et l'état de soudure des os longs.

Le premier stade observé à Cagny-l'Épinette correspond à des individus très jeunes dont les premières molaires sont des bourgeons non usés (stade 1 de Frison) (Brugal et David, 1993). Les deuxièmes molaires sont des bourgeons inclus. Les maxillaires correspondant à ce stade montrent une D3 non usée, la première molaire n'est pas encore sortie. II s'agit d'animaux de moins de 6 mois (Richter, 1982).

Le deuxième stade est très bien représenté par 11 hémi-mandibules soit un minimum de 5 individus dans la couche 11 et 1 dans le niveau IJ. Les incisives lactéales sont fonctionnelles, la première incisive est incluse. Les D2, D3 sont usées et la D4 est fonctionnelle, la couronne n'est pas altérée. La première molaire définitive est en fonction. La hauteur de la couronne est encore presqu'entière. La M2 n'est pas sortie. L'allure du protoconide donne un âge de moins de 18 mois. Sur les maxillaires, les D4 et M1 sont juste usées, mais la M2 est incluse.

Le troisième stade est bien représenté : un individu dans les niveaux $\mathrm{H}, \mathrm{I}, \mathrm{I} 2$ et $\mathrm{J}$ : la première molaire est déjà usée, le fût de cette dent a diminué de moitié, la seconde molaire est bien fonctionnelle, la première incisive est usée. Sur les maxillaires correspondant à ce degré d'usure, la D4 est prête à tomber, les prémolaires définitives ne sont pas usées. $\mathrm{Ce}$ stade correspond à un âge d'environ 30 mois.

Le quatrième stade est représenté par des dents inférieures isolées, la dernière molaire est fonctionnelle. II s'agit d'une dent de plus de 5 ans. Pour ce stade les maxillaires sont mieux fournis, les prémolaires sont usées sauf la P4 qui est juste fonctionnelle.

L'avant-dernier stade, représenté dans la couche 11 correspond à des adultes dont les prémolaires sont bien usées, le fût de la deuxième molaire est réduit de moitié et la dernière molaire présente une usure prononcée. D'après Russell, il peut s'agir de bêtes de moins de 9 ans.
Pour le dernier stade, la dernière molaire est très usée, ce qui correspond à des animaux de près de 15 ans. Le maxillaire observé dans le niveau 11 montre effectivement des prémolaires très usées, les dernières molaires sont arasées.

La formule de Klein-Spinage n'a pas été utilisée, bien que la hauteur des métacones non usés soit connue, car le nombre de dents adultes est trop faible.

Le squelette post-céphalique des jeunes animaux a pu être analysé. En général, les os non soudés présentent trois groupes de taille que nous essaierons de rapprocher des stades dentaires définis. Les épiphyses ne se soudent pas toutes en même temps, la chronologie de ces soudures est bien établie pour les animaux domestiques mais pas pour les aurochs, il est donc intéressant, sur le site de Cagny-l'Épinette, de proposer un tableau pour cette espèce. On considère actuellement que les grands bovidés atteignent leur taille adulte vers 2,5 ans. Les ossements ne sont pas tous soudés à cette époque puisque les plus tardifs se font vers 4 ans et plus. Les bêtes de 3 ans sont grandes, voire plus grandes que les adultes, les os "se tassent" plus tard.

Pour les humérus, la hauteur de la diaphyse au stade 3 correspond à la taille adulte. Dans ce cas la soudure médiale distale est réalisée mais pas les soudures postérieures. Les épiphyses proximales ne sont pas soudées. La soudure distale est réalisée vers 20 mois. L'animal a donc entre 20 et 48 mois.

II semble donc bien que le stade 2 correspond au deuxième groupe évoqué par l'éruption dentaire et attribué à des animaux de moins de 18 mois. Le premier groupe est attribué à des veaux de 6 mois. Sur les radius, l'épiphyse proximale est soudée vers 15 mois, la seconde au cours de la quatrième année. Le petit fémur est à rapprocher des individus de 6 mois.

Tabl. 12 - Longueur des diaphyses des ossements de jeunes aurochs, Cagny-l'Épinette.

\begin{tabular}{|c|c|c|c|c|c|c|c|}
\hline âge & humérus & radius & fémur & tibia & métatarse & $\begin{array}{c}\text { première } \\
\text { phalange }\end{array}$ & $\begin{array}{c}\text { deuxième } \\
\text { phalange }\end{array}$ \\
\hline stade 1 & 180 & & 230 & & & 53 & 33 \\
\hline stade 2 & 220 & 250 & 270 & 272 & 215 & $61-64$ & 37 \\
\hline stade 3 & 230 & 260 & & 305 & 260 & & \\
\hline
\end{tabular}

Le troisième stade de taille du tibia est très proche de la taille adulte sans en posséder la robustesse. La soudure de l'extrémité distale s'effectue entre deux ans et deux ans et demi et ces os peuvent correspondre à cet âge.

Les carpiens et tarsiens présentent également trois catégories. Le dernier stade correspond à la taille adulte, mais l'os est plus gracile et moins dense. On remarque que le talus atteint rapidement sa taille adulte mais que la largeur distale augmente progressivement.

Le stade 2 des premières phalanges correspond à une hauteur totale de la phalange de $75 \mathrm{~mm}$, les bords sont presque soudés sur certains spécimens. Cette soudure intervient vers 20 mois. La présence de phalanges adultes en plus grand nombre s'explique bien par leur soudure précoce.

Les deuxièmes phalanges sont également fréquentes. Le deuxième lot correspond à des phalanges proches de la taille adulte $(\mathrm{Ht}$ totale $=$ 47). II s'agit d'individus d'un âge compris entre 15 et 18 mois. Cette soudure encore plus précoce permet de bien dater le lot d'ossements non soudés le plus important, particulièrement dans le niveau 11.

Les catégories de tailles bien différenciées nous permettent de donner un âge précis à la plupart des ossements de jeunes bovidés : les os longs des bêtes de 6 mois sont plus rares et probablement moins bien conservés tandis que ceux des individus de 18 mois sont nombreux. Les os du stade 3 correspondent à des animaux de deux ans et demi environ.

L'allure de la population des cervidés est décrite d'après les bois, bois de massacres, qui indiquent la présence de mâles et donnent une idée de leur âge. Les dents sont attribuées selon leur nature et leur usure à une catégorie jeune, adulte ou adulte âgé. 
L'âge des chevaux est particulièrement bien documenté d'après l'usure des incisives et des dents jugales dans les traités vétérinaires. Les ossements d'équidés étant très rares, il sera simplement signalé si les os sont soudés ou non. On peut remarquer que par ce mode de calcul, les individus adultes sont probablement sous-estimés car ils ne peuvent être aussi bien différenciés que les jeunes.

Le niveau $\mathrm{H}$ comprend au moins trois aurochs, un très jeune de près de 6 mois, un individu adulte de plus de 4 ans et une bête plus âgée. Les cerfs sont au minimum deux individus, un jeune, dont la molaire supérieure est en bourgeon, et un adulte. Tous les restes de chevaux peuvent être attribués à un seul individu, un adulte jeune entre 4 et 5 ans.

Le niveau I comprend un veau de 6 mois, deux adultes jeunes et un adulte très âgé. Les cerfs sont au moins trois individus dont un jeune et deux jeunes adultes. Les bois de massacre attestent la présence de deux mâles. Les dents peu usées permettent de comprendre la petite taille des bases de bois. Le cheval est représenté par un adulte.

Le niveau 11 comprend au moins un veau de 6 mois, trois de 18 mois, deux jeunes aurochs de moins de 36 mois et deux adultes. Les cervidés comptent au moins un jeune de deuxième année et deux adultes. Le daim est également représenté par un jeune et un adulte. Un cheval adulte complète ce niveau.

Le niveau IJ a livré un jeune aurochs de 18 mois dont il reste la plupart des ossements, et un adulte de plus de 5 ans, un jeune cerf de deux ans et deux chevaux, un jeune de moins de deux ans et un de trois ans.

Le niveau $\mathrm{J}$ comprend un jeune aurochs de 18 mois, deux jeunes de 30 mois et un adulte. Les cerfs sont au nombre minimum de trois, un jeune adulte et deux adultes dont l'un plus âgé. On doit également noter la présence d'au moins un mâle parmi eux. II y a un jeune poulain de 6 mois et un cheval adulte.

Cet inventaire permet de comprendre l'importance des jeunes bovidés dans ce gisement. II convient de savoir si leur apport sur le site a bien la même origine que les autres animaux.

On peut noter que les individus de 18 mois caractérisent les niveaux 11 , IJ et $\mathrm{J}$. Ces niveaux archéologiques présentent également de nombreuses similitudes quant à l'apport des bois de chute et à la conservation en général des ossements (tabl. 13).

\section{- Les activités anthropiques révélées par les ossements}

Surtout mises en évidence pour le niveau 11 , elles sont principalement de deux types:

- le dépeçage des animaux, la décarnisation et les désarticulations, ainsi que la fracturation volontaire des ossements,

\section{- l'utilisation des ossements.}

L'importance primordiale des ossements sur le site de Cagny-l'Épinette semble bien liée à des activités de subsistance. La plupart des ossements adultes d'aurochs, de cerfs et de chevaux portent des traces du premier type, correspondant à des activités de boucherie et de consommation de la mœlle. Les schémas présentés regroupent les traces observées sur les différents sols pour chacune de ces trois espèces (fig. 15 à 18).

Les stries prises en compte sont les stries les plus caractéristiques, visibles à l'œil nu, ayant pour origine l'emploi d'un outil pointu et coupant. Les stries observées sur le crâne, les apophyses des vertèbres, les côtes sont attribuées au décharnement des carcasses.

Nous n'avons pu isoler les stries de désarticulation proprement dite. Les stries très épaisses, courtes et souvent multiples, au niveau de l'extrémité proximale des métapodes de cerf et d'aurochs, sont plutôt à mettre en relation avec le décollement des tendons extenseurs du pied.

La fracturation la plus fréquente est une fracturation longitudinale, pour les métapodes, fémurs, tibias et radius. Les encoches consécutives à la percussion sont visibles sur les os robustes de cheval et d'aurochs. Ils sont rarement doubles. La localisation de ces impacts est comparable à celles observées sur d'autres sites du Paléolithique inférieur (Caune de l'Arago, Orgnac 3, Torralba).

L'humérus de cheval (Ép. $9020 \mathrm{~V}$ $1150)$ présente une plage de piquetage sur la diaphyse distale interne (fig. 17). La zone d'altération s'étend sur une surface de $10 \times 3 \mathrm{~cm}$. II semble que cette plage osseuse ait servi d'enclume. L'articulation distale de cet os présente une "usure" altérant la face distale de la trochlée interne. Cette altération est circulaire et semble également issue d'un piquetage important. Le même type

Tabl. 13 - Inventaire des restes osseux de Cagny-l'Épinette.

\begin{tabular}{|c|c|c|c|c|c|c|c|c|c|c|c|c|c|c|c|c|c|c|}
\hline & $\mathrm{F} 1$ & $\mathrm{~F} 2$ & $\mathrm{G}$ & G1 & $\mathrm{Gx}$ & $\mathrm{H}$ & $\mathrm{H} 1$ & $\mathrm{Hx}$ & 1 & 10 & 11 & $11 \mathrm{~b}$ & $11 \mathrm{~J}$ & 12 & IJ & $\mathrm{J}$ & J1 & Ensemble \\
\hline Bos & 0 & 1 & 1 & 0 & 0 & 36 & 0 & 0 & 42 & 1 & 521 & 1 & 16 & 2 & 39 & 94 & 0 & 759 \\
\hline carnivores & 0 & 0 & 4 & 0 & 0 & 0 & 0 & 0 & 0 & 0 & 2 & 0 & 0 & 0 & 0 & 0 & 0 & 2 \\
\hline Cervus & 1 & 0 & 4 & 0 & 0 & 23 & 4 & 1 & 46 & 6 & 102 & 0 & 0 & 4 & 20 & 57 & 1 & 269 \\
\hline Crocuta & 0 & 0 & 0 & 0 & 0 & 0 & 0 & 0 & 1 & 0 & 0 & 0 & 0 & 0 & 0 & 0 & 1 & 1 \\
\hline Dama & 0 & 0 & 0 & 0 & 0 & 0 & 1 & 0 & 1 & 0 & 8 & 0 & 0 & 0 & 1 & 0 & 0 & 11 \\
\hline Elephas & 0 & 0 & 0 & 0 & 0 & 1 & 0 & 0 & 0 & 0 & 0 & 0 & 0 & 0 & 0 & 0 & 0 & 1 \\
\hline $\begin{array}{l}\text { Equus } \\
\text { grds } \\
\text { herbivores }\end{array}$ & 0 & 2 & 0 & 1 & 0 & 15 & 3 & 0 & 6 & 0 & 14 & 0 & 0 & 1 & 6 & 5 & 0 & 53 \\
\hline indéterminés & 0 & 0 & 0 & 0 & 2 & 6 & 0 & 0 & 2 & 0 & 8 & 0 & 0 & 1 & 3 & 1 & 0 & 23 \\
\hline $\begin{array}{l}\text { indétermés } \\
\text { petits } \\
\text { herbivores }\end{array}$ & 0 & 0 & 3 & 0 & 0 & 44 & 3 & 0 & 42 & 0 & 215 & 0 & 4 & 3 & 18 & 24 & 0 & 326 \\
\hline indéterminés & 0 & 0 & 0 & 0 & 0 & 2 & 1 & 0 & 3 & 0 & 15 & 0 & 0 & 0 & 1 & 0 & 0 & 22 \\
\hline Vulpes & 0 & 0 & 0 & 0 & 0 & 0 & 0 & 0 & 0 & 0 & 1 & 0 & 0 & 0 & 0 & 0 & 0 & 1 \\
\hline Ensemble & 1 & 3 & 8 & 1 & 2 & 127 & 12 & 1 & 143 & 7 & 889 & 1 & 20 & 11 & 88 & 181 & 1 & 1501 \\
\hline
\end{tabular}




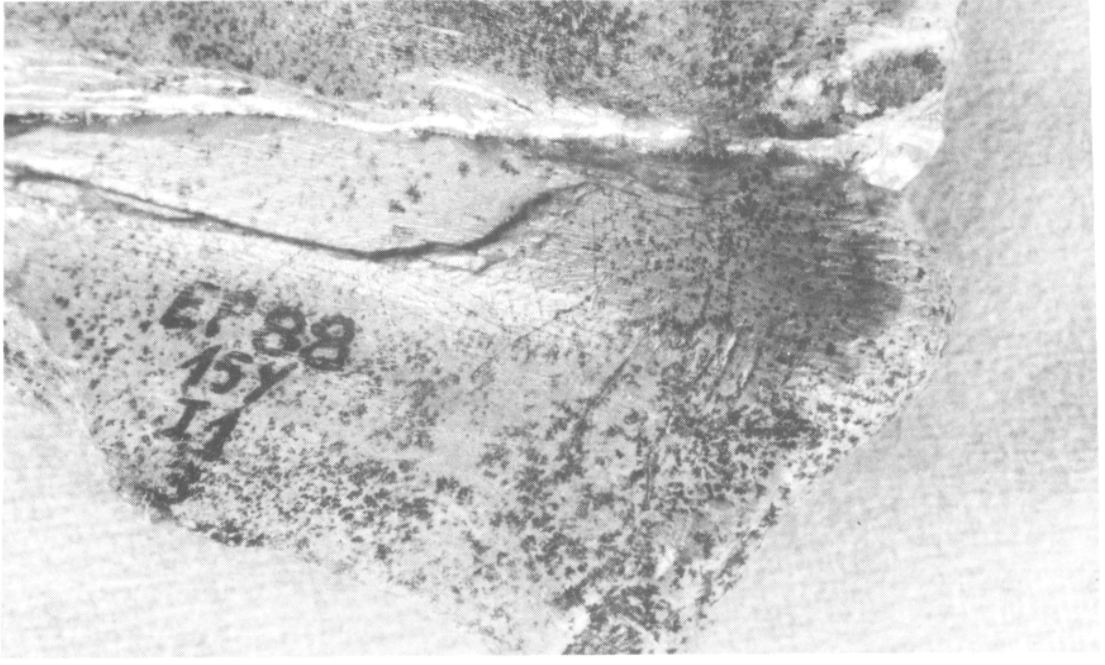

Fig. 15 - Cagny-l'Épinette, niveau I1. Stries courtes et parallèles sur l'extrémité proximale antérieure du métatarsien de cerf, Ép. 88, 15 Y, 11,3.

d'altération a également été noté sur les os longs adultes d'aurochs: un bassin, deux diaphyses d'humérus, un métacarpe et un métatarse. Toutes ces zones de piquetage ont été observées dans le niveau 11 où l'état de surface des ossements est particulièrement bien conservé. Nous n'avons pour l'instant pas retenu d'interprétation pour les traces de ce genre.

Un radius de cerf (Ép. $9017 \mathrm{VI1} 4$ ) présente une fracture en biseau au tiers distal. L'extrémité de ce biseau est polie. II n'a pas été enregistré de polis sur aucun des ossements du gisement et cette exception très localisée sur l'os mérite une étude particulière du type de poli.

Les bois de chutes concentrés sur le site dans les niveaux $\mid 1$, IJ et $\mathrm{J}$ ont été apportés volontairement. Ces bois n'ont pas été transformés. Un seul andouiller a été arraché.

Les observations relatives à la présence d'ossements comme les bois de chute et de traces d'utilisation sur les os du niveau 11 nous invitent à envisager une activité humaine peut-être plus élaborée que ce qui pouvait apparaître jusqu'à présent.

En conclusion, les diverses espèces rencontrées à Cagny-l'Épinette correspondent à des espèces du Pléistocène moyen : Le cheval se caractérise par sa grande taille, le rapprochant d'Equus mosbachensis, de Equus achenheimensis et de Equus ferus d'Hoxne et par sa gracilité relative. Le cerf est petit par rapport aux gisements les plus récents du Pléistocène moyen. L'aurochs, de forte taille, se classe parmi les popu- lations de Bos primigenius trochoceros. Cagny-l'Épinette est un gisement bien daté stratigraphiquement qui permet une meilleure connaissance de ces trois espèces prépondérantes dans les sites préhistoriques de cette époque.

L'environnement décrit d'après les analyses polliniques (Munaut, 1988 et ci-dessus) concorde bien avec les espèces animales observées. Le daim, le cheval hydruntin et l'éléphant antique sont généralement admis dans des biotopes tempérés. La situation du gisement en bord de rivière paraît tout indiquée pour comprendre la présence des aurochs qui recherchent plutôt les milieux humides.

La conservation des ossements varie d'un niveau à l'autre. Les niveaux $F, G$ et $H$ ne permettent pas une étude archéozoologique pous- sée car le matériel disparu est très important.

Les niveaux inférieurs, surtout 11 et IJ, montrent d'excellentes conditions de conservation. L'état de surface des ossements est très propice à l'étude des stries : il y a peu d'intervenants extérieurs aux hommes sur le site et le transport post-dépositionnel est nul. Les traces de rongeurs, carnivores et charriage représentent $0,01 \%$ du matériel pour 11 . La fracturation secondaire est aisément identifiable. L'enfouissement du niveau 11 s'est déroulé très rapidement et l'occupation des hommes elle-même fut probablement de courte durée.

Sur le niveau $\mathrm{J}$, les interventions autres que celles de l'homme sont un peu plus fréquentes. Les traces superficielles dues aux carnivores et aux rongeurs affectent $0,04 \%$ du total des ossements. Ce niveau, probablement très étendu permet de mieux comprendre les interactions entre l'homme et le milieu, la surface d'occupation est restée découverte plus longtemps et sûrement plus longtemps exploitée.

Ces trois niveaux comptent de nombreux bovidés âgés d'environ 6 , 18 et 30 mois. En admettant que les naissances se font vers la fin du mois de mai, on peut situer ces accumulations vers le début de l'hiver. La présence de bois de chute de jeunes cerfs permet également d'estimer leur ramassage vers cette époque.

Les fouilles qui continuent sur ce site permettront de mieux comprendre le comportement différentiel des hommes par rapport aux car-

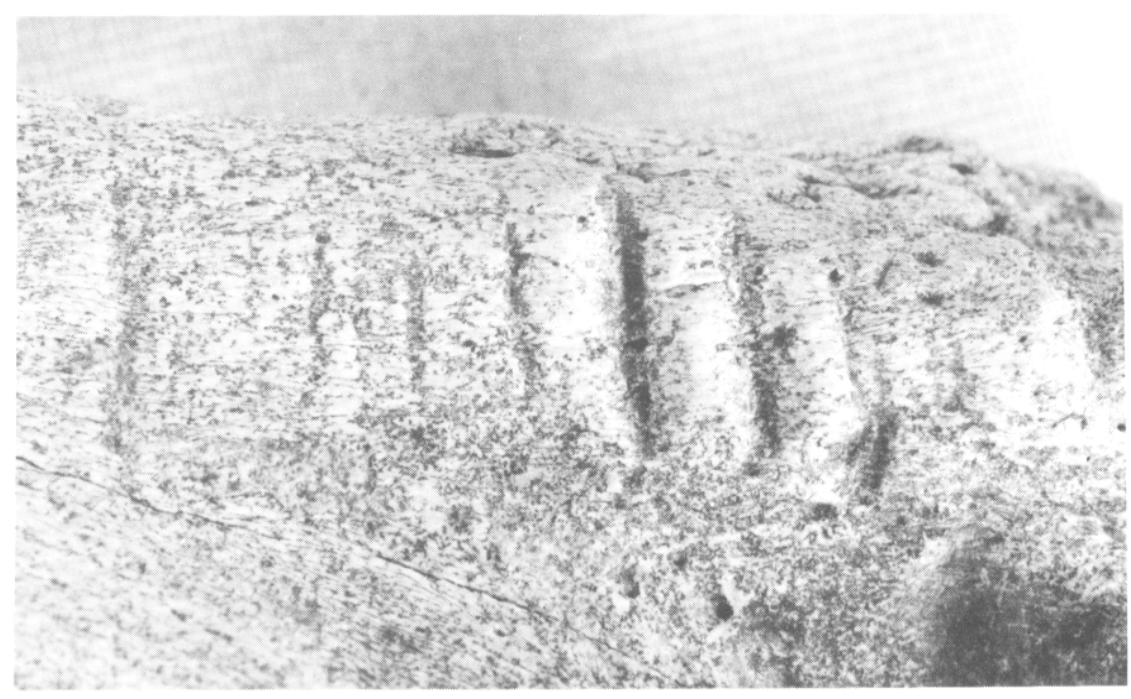

Fig. 16 - Cagny-l'Épinette, niveau 11. Stries courtes et parallèles sur l'extrémité proximale postérieure du métatarsien d'aurochs, Ép. 90, 20 Q, I1,25. 
casses d'adultes et carcasses de jeunes aurochs. La découverte de nouvelles pièces osseuses portant des plages de piquetage devrait enrichir la connaissance des os utilisés par l'homme pour cette période.

\section{CONCLUSION}

La fouille du gisement acheuléen de Cagny-l'Épinette apporte de nombreuses données concernant le contexte sédimentaire et environnemental des occupations acheuléennes dans le bassin de la Somme. Le contexte chronostratigraphique, les datations absolues, et le degré d'évolution de la grande faune permettent d'attribuer les niveaux archéologiques de la séquence fluviatile fine à la fin de l'étage isotopique 10 et à l'étage 9. Cette interprétation est toutefois en contradiction avec les résultats des analyses concernant les micromammifères. La poursuite du programme d'étude des micromammifères, qui doit être étendu aux dépôts des différentes nappes alluviales du bassin de la Somme, est susceptible d'apporter une explication à cette contradiction apparente. Comme cela a pu être observé dans d'autres gisements, les conditions climatiques, de type continental, s'inscrivent dans des ambiances de type Tardiglaciaire à Début Glaciaire avec un paysage de steppe arborée.

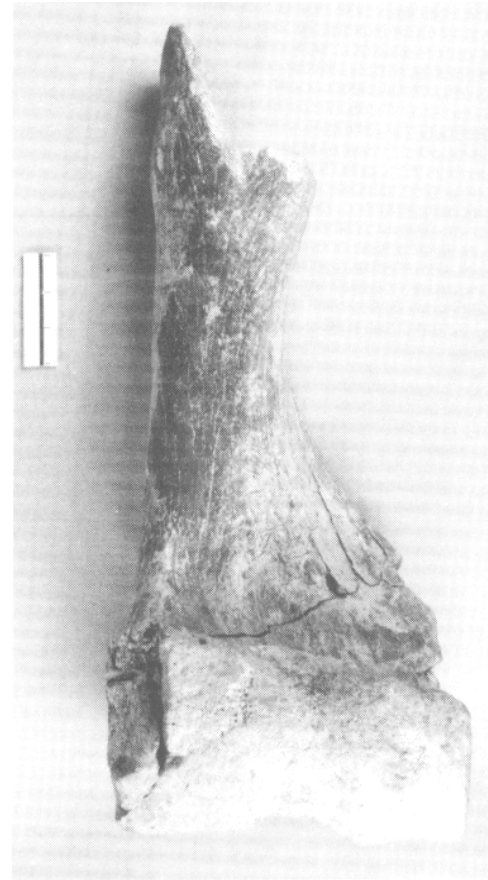

Fig. 17 - Cagny-l'Épinette, niveau I1. Zone de piquetage sur la trochée distale de l'humérus de cheval, Ép. 90, $20 \mathrm{~V}, 11,50$.

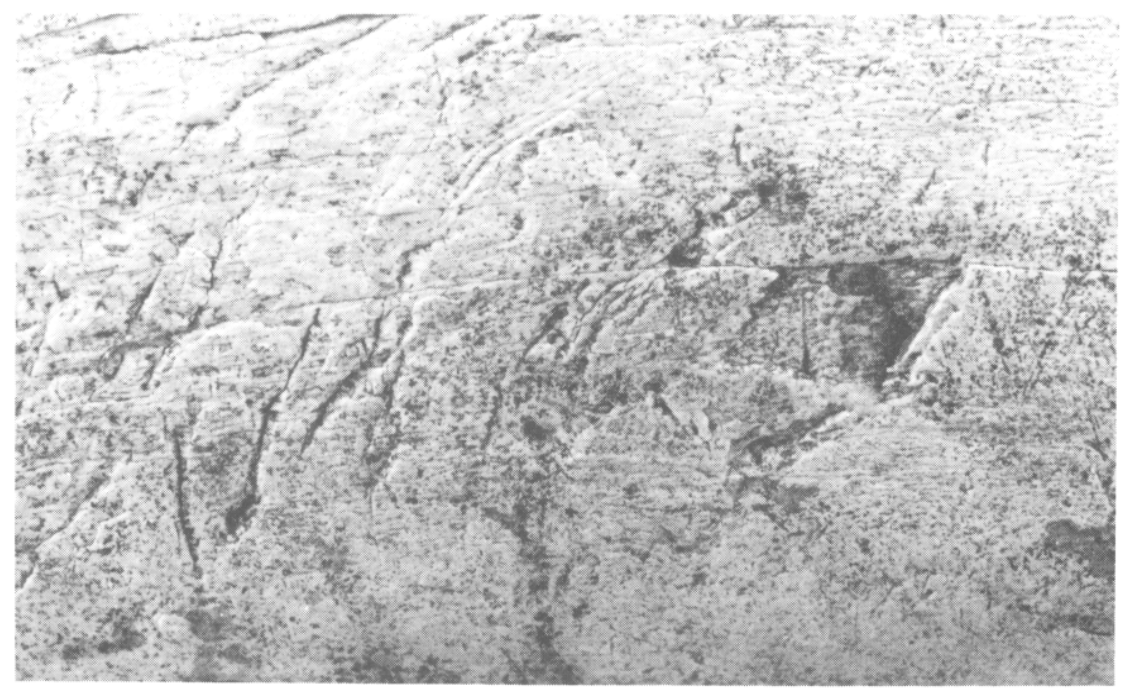

Fig. 18 - Cagny-l’Épinette, niveau I1. Plage de piquetage sur la diaphyse du métatarsien d'aurochs, Ép. 90, 20 Q, 11,25.

L'un des intérêts majeurs du gisement de Cagny-l'Épinette résulte de la présence d'ossements d'animaux montrant des liens avec des activités anthropiques. La poursuite de l'étude spatiale de plusieurs niveaux $(H, I-J, 11)$ permettra de mieux cerner la part des différents facteurs qui interviennent dans leur présence (collecte de carcasses et exploitation par les hommes, homogénéité ou hétérogénéité des niveaux, impact de la dynamique du cours d'eau, problèmes taphonomiques) et qui peuvent varier d'un niveau à l'autre. D'ores et déjà, il est possible de constater qu'il existe dans les séquences sédimentaires des nappes de la "moyenne" terrasse de la Somme des témoignages correspondant à des activités spécialisées : collecte de la matière première et présence d'ateliers (façonnage de bifaces et production d'éclats) dans certains niveaux de Cagny-la Garenne, exploitation de la grande faune à Cagny-l'Épinette avec un comportement plutôt opportuniste pour les industries lithiques.

Les recherches menées à Cagny-l'Épinette ont bénéficié du soutien financier du Ministère de la Culture (Sous-direction de l'archéologie), du C.N.R.S. (A.T.P. archéologie métropolitaine), du Conseil Général de la Somme, de l'Université des Sciences et Technologies de Lille, de la National Science Foundation, ainsi que du support logistique du Conseil Général du Pas-de-Calais (Musée départemental de Préhistoire, Arras).

\section{Bibliographie}

ANTOINE P. (1990) - Chronostratigraphie et environnement du Paléolithique du bassin de la Somme. Publications du C.E.R.P., $\mathrm{n}^{\circ} 2,231$ p., 187 fig., 1 carte coul., 16 photos.

Antoine P. et Tuffreau A. (1993) Contexte stratigraphique, climatique et paléotopographique des occupations acheuléennes de la moyenne terrasse de la Somme. Bull. Soc. Préhist. Fr., 90 , p. 243-250, 7 fig.

Aouraghe H. (1992) - Les faunes de grands mammifères du site pléistocène moyen d'Orgnac 3 (Ardèche, France). Thèse de Doct., Museum Nat. Hist. Nat., Paris, 495 p.

BALESCU S. (1988) - Apports de la thermoluminescence à la stratigraphie et à la sédimentologie des loss de l'Europe du Nord-Ouest. Thèse Doct. Scien. Univ. Libre Bruxelles, vol. I: 199 p., 104 fig., 13 tabl. ; vol. II : annexes, 144 p., 8 fig., 20 tabl.

Barral L. et Simone S. (1972) - Le Mindel-Riss et le Riss à la grotte d'Aldène (Cesseras, Hérault). Bull. Musée Anthr. Préhist. Monaco, 18, p. 45-68.

BAtes M. R. (1993) - Quaternary Aminostratigraphy in Northwestern France. Quaternary Science Reviews, 12, p. 793-809, 9 fig.

BoRdes F. (1980 a) - Question de contemporanéité : l'illusion des remontages. Bull. Soc. Préhist. Fr., 77, p. $132-133,1$ fig.

BORDES F. (1980 b) - Savez-vous remonter les cailloux à la mode de chez nous ? Bull. Soc. Préhist. Fr., 77 , p. 232-234.

BrugaL J.P. (1984) - Le Bos primigenius Boj. 1827 du Pléistocène moyen des grottes de Lunel-Viel (Hérault). Bull. Musée anthropol. Monaco, $\mathrm{n}^{\circ} 28$, 1984-1985, p 7-62.

BRUGAL J.P. et DAVID F. (1993) - Usure dentaire, courbe de mortalité et "saisonnalité" : Les gisements du Paléolithique moyen à grands bovidés, Exploitation des animaux sauvages à travers le temps. Ed. A.P.D.C.A., Juans-les-Pins, p 63-77. 
BUNN H.T. (1981) - Archaeological evidence for meat-eating by Plio-Pleistocene hominids from Koobi Fora and Olduvai. Nature, 291, p. 574-577.

BUNN H.T. (1983) - Evidence on the diet and subsistence patterns of Plio-Pleistocene hominids at Koobi Fora, Kenya, and Olduvai Gorge, Tanzania. In : J. Clutton-Brock and C. Grigson eds. Animals and Archaeology: 1 Hunters and Their Prey. B.A.R. International Series, 163, Oxford, p. 21-30.

BUNN H.T. (1986) - Patterns of skeletal representation and hominid subsistence activities at Olduvai Gorge, Tanzania, and Koobi Fora, Kenya. Journal of Human Evolution, 15, p. 673-90.

BUNn H.T. (1989) - Diagnosing PlioPleistocene hominid activity with bone fracture evidence. In : R. Bonnichsen and M.H. Sorg, eds. Bone Modification. Institute for Quaternary Studies, University of Maine, Orono.

BunN H.T. et Kroll E.M. (1986) - Systematic butchery by Plio-Pleistocene hominids at Olduvai Gorge, Tanzania Current Anthropology, 27, p. 431-452.

Cahen D., Keeley L. et Van noten F. (1979) - Stone tools, toolkits and human behaviour in prehistory. Current Anthropology, 20, p. 661-683.

Chase P. et Dibble H. (1987) - Middle Paleolithic Symbolism: A Review of Current Evidence and Interpretations. Journal of Anthropological Archaeology, 6, p. 263-296.

Chase P., Armand D., Debenath A. Dibble $H$. et Jelinek A. (1994) - Taphonomy and Multiple Hypothesis Testing: An Object Lesson from the Mousterian. Journal of Field Archaeology, in press.

Corbet G.B., HarRIS S., ed. (1991) - The Handbook of British Mammals. 3rd edit. Blackwell Scientific Publications, Oxford.

CoRDY J.-M. (1989) - Les micromammifères de Cagny-l'Épinette (rapport préliminaire). In : Livret-guide de l'excursion dans la vallée de la Somme. Colloque "L'Acheuléen dans l'Ouest de l'Europe" Saint-Riquier, p. 80-81.

DESCOMBES J.-C. (1983) - Étude paléontologique du gisement pléistocène moyen de Tourville-la-Rivière (SeineMaritime). Bull. Ass. Fr. Et. Quatern. 1983-4, p 161-169.

Dibble H. (1987) - Measurement of Artifact Provenience with an Electronic Theodolite. Journal of Field Archaeology, 14, p. 249-254.

Dibble H. et Rolland N. (1992) - On assemblage variability in the Middle $\mathrm{Pa}$ leolithic of Western Europe: History, Perspectives and a New synthesis. In The Middle Paleolithic: Adaptation, Behavior and Variability, edited by $H$. Dibble and P. Mellars. University Museum Press, Symposium Series, Philadelphia, 2, p. 1-28, 5 fig.

Dibele H. et McPherRon S. (1988) - On the computerization of Archaeological Projects. Journal of Field Archaeology, 15 , p. $431-440,2$ fig.

DibBle $H$. et Lenoir $M$. (in press) - The Middle Paleolithic Site of Combe-Capelle Bas (France), edited by $\mathrm{H}$. Dibble and $M$. Lenoir. University Museum, University of Pennsylvania.
EISENMANN V. (1991) - Les chevaux quaternaires européens (Mammalia, Perrissodactyla). Taille, typologie, biostratigraphie et taxonomie. Géobios, $n^{\circ} 24$, fasc. 6, p. 747-759.

ENLoE J. et DAVID F. (1989) - Le remontage des os par individus: le partage du Renne chez les Magdaléniens de Pincevent (La Grade Paroisse, Seineet-Marne). Bull. Soc. Préhist. Fr., 86, p. 275-281.

Freeman L.G. (1975) - Acheulean sites and stratigraphy in Iberia and the Maghreb. After the Australopithecines (K.W. Butzer and G.LI. Isaac, eds), Mouton, The Hague, p. 661-744.

Freeman L.G. (1978) - The analysis of some occupation floor distributions from earlier and Middle Paleolithic sites in Spain. Views of the Past (L.G. Freeman, ed), Mouton. The Hague, p. 57-116.

GUADELLI J.-L. (1987) - Contribution à l'étude des zoocénoses préhistoriques en Aquitaine (Wurm ancien et interstade wurmien). Thèse doct., Université de Bordeaux I, $3 \mathrm{t}$.

Haesaerts P. et Dupuis Ch. (1986) Contribution à la stratigraphie des nappes alluviales de la Somme et de l'Avre dans la région d'Amiens. In : "Chronostratigraphie et faciès culturels du Paléolithique inférieur et moyen dans l'Europe du Nord-Ouest". Suppl. Bull. Ass. Fr. Et. Quatern., 26, p. 171186, 7 fig.

Haesaerts P., Balescu S., Dupuis Ch. et VAN VIIET-LANOË B. (1984) - Contribution à la stratigraphie des gisements paléolithiques de Cagny (Somme). Cahiers de géographie physique, Lille, 5 , p. 77-94, 6 fig.

HEINRICH W.D. (1978) - Zur biometrischen Erfassung eines Evolutionstrends bei Arvicola (Rodentia, Mammalia) aus dem Pleistozän Thüringens. Säugetlerkdl. Inform., 2, Berlin, p. 3-21, 5 fig.

HEINRICH W.D. (1982) - Zur Evolution und Biostratigraphie von Arvicola (Rodentia, Mammalia im Pleistozän Europas. Z. geol. Wiss., Berlin, 10, 6, p. 683735.

HeinRich W.D. (1987) - Neue Ergebnisse zur Evolution und Biostratigraphie von Arvicola (Rodentia, Mammalia) im Quartär Europas. Z. geol. Wiss., 15, 3 , Berlin, p. 389-406, 9 fig.

Hofman J.L. (1992) - Putting the Pieces Together : an Introduction to Refitting. In: Piecing together the Past : Applications of Refiting Studies in Archaeology (J.L. Hofman and J.G. Enloe, ed.). $B A R$ Intern. Series, 578 , p. 1-20.

ISAAC G.L. (1967) - Towards the interpretation of occupation debris: some experiments and observations. Kroeber Anthropological Society Papers, 37, p. 31-57, 8 fig

ISAAC G.L. (1983) - Bones in contention: competing explanations for the juxtaposition of Early Pleistocene artifacts and faunal remains. In : Animals and Archaeology (J. Clutton-Brock and C. Grigson, eds.), BAR Intern. Series, 163, Oxford, p. 3-20, 6 fig.

ISAAC G.L. (1984) - The archaeology of human origins: studies of the Lower Pleistocene in East Africa, 1971-1981.
Advances in Old World Archaeology (F. Wendorf, ed.), vol. 3, Academic Press. Orlando, p. 1-87, 14 fig.

KeLling G. et Williams P.F. (1967) Flume studies of the reorientation of pebbles and shells. Journal of Geology, 75, p. 243-267.

KIND C.J. (1985) - Die Verteilung von Steinartefakten in Grabungsfläschen. Urgeschichtliche Materialhefte, 7, Tübingen, 283 p., 181 fig., 32 tabl.

KOLFSCHOTEN T. V. (1990) - The evolution of the mammal fauna in the Netherlands and the middle Rhine Area (Western Germany) during the late Middle Pleistocene. Meded. Rijks Geol. Dienst, 43-3, p. 1-69, 27 fig.

KLUSKENS S. (sous presse) - Archaeological Taphonomy of Combe-Capelle Bas from Artifact Orientation and Density Analysis. In : The Middle Paleolithic Site of Combe-Capelle Bas (France) edited by $H$. Dibble and $M$. Lenoir. University Museum, University of Pennsylvania, Philadelphia.

LAMOTTE A. (1994) - Les industries à bifaces du Pléistocène moyen dans l'Europe du Nord-Ouest : données nouvelles des gisements du bassin de l'Escaut, de la Somme et de la baie de Saint-Brieuc. Thèse Doct. Univ. Sciences Techn. Lille, 382 p., 101 fig.

LAURENT M. (1993) - Datation par résonance de spin électronique (E.S.R.) de quartz de formations quaternaires : comparaison avec le paléomagnétisme. Thèse Doct., Museum Nat. Hist. Nat., Paris, 103 p., illustr.

LÉOPOLD J. (1993) - Les industries lithiques de Cagny-L'Épinette, Cagny Cimetière et Cagny-La-Garenne (Somme) dans le contexte Nord-Ouest Européen. Thèse, Inst. Paléont. Hum., 461 p., 488 fig.

LEROI-GOURHAN A. (1961) - Les fouilles d'Arcy-sur-Cure. Gallia-Préhistoire, IV, p. 3-16, 8 fig.

LeROI-GourHAN A et BrÉZILLON M. (1972) - Fouilles de Pincevent. Essai d'analyse ethnographique d'un habitat magdalénien. Suppl. Gallia-Préhistoire, VII, 331 p., 199 fig., plans h.-t.

LOVEJOY C.O. (1981) - The origin of man. Science, 211, p. 341-350.

LUMLEY H. de, dir. (1969) - Une Cabanne acheuléenne dans la grotte du Lazaret. Mém. Soc. Préhist. Fr., 7, 235 p., illustr.

LUMLEY H. de (1972) — La grotte de l'Hortus (Valflaunès, Hérault). Études Quaternaires, mém. $n^{\circ} 1$, Université de Provence, 668 p., illustr.

LUMLEY H. de et BOone Y. (1976) - Les structures d'habitat au Paléolithique inférieur. La Préhistoire Française I, 1, p. 625-643, 22 fig.

MANIA D. et WEBER T. (1986) - Bilzingsleben III. Veröffentlichungen. Landesmuseums für Vorgeschichte in Halle, 39.

MellaRS P. (1988) - The Origins and Dispersal of Modern Humans. Current Anthropology 29, p. 186.

Mellars P. (1991) - Cognitive Changes and the Emergence of Modern Humans in Europe. Cambridge Archaeological Journal, 1, p. 63-76.

Moigne A.-M. (1988) - Les faunes du gisement de Cagny-l'Épinette. Actes du 
Colloque "Cultures et Industries paléolithiques en milieu lœssique", Amiens, 9-11 déc. 1986. Rev. archéol. Picardie, $n^{\circ} 1-2$, p. 69-73.

Moigne A.-M. (1989) - Taphonomie des gisements paléolithiques. Le Temps de la Préhistoire (J.-P. Mohen, dir.), I, Paris, p. 140-141.

Munaut A.-V. (1988) - L'environnement végétal de quelques dépôts quaternaires du bassin de la Somme (France). Actes du Colloque "Cultures et Industries paléolithiques en milieu lœssique", Amiens, 9-11 déc. 1986. Rev. archéol. Picardie, $n^{\circ} 1-2$, p. 4555,10 fig.

PotTs R.B. (1984) - Hominid hunters? Problems of identifying the earliest hunter/gatherers. Hominid Evolution and Community Ecology (R. Foley, ed.), Academic Press, New York, p. 129-166

PRAT F. (1968) - Recherches sur les Équidés pléistocènes en France. Thèse de doctorat d'État, Univ. Bordeaux I, $696 \mathrm{p}$.

RICHTER J. (1982) - Adult and juvenile Aurochs, Bos primigenius Boj. from the Maglemosian Site of Ulkestrup Lyng Ost, Denmark. Journal of Archeological Science, 9, p. 247-259.

SERrE F. (1993) - Les grands herbivores de la grotte du Lazaret, Nice, AlpesMaritimes. Thèse Doct., Museum Nat. Hist. Nat., Paris, 1993, 480 p.

SIMEK J. (1987) - Spatial order and behavioural change in the French Paleolithic. Antiquity, 61, p. 25-40.

Stekelis M., BAR-Yosef O. et Schick T. (1969) - Archaeological Excavations at Ubeidiya, 1964-1966. Israel Academy of Sciences and Humanities. Jerusalem.

Stopp M. (1993) - Taphonomic Analysis of the faunal assemblage. The Lower Paleolithic site at Hoxne, England (R. Singer, B.G. Gladfelter and J.J. Wymer, eds), Univ. of Chicago Press, p. 138-149.

StuARt A.J., WOlfF R.G., LISTER A.M., SINGER R. et EGGINTON J.M. (1993) - Fossil Vertebrates. The Lower Paleolithic site at Hoxne, England (R. Singer, B.G. Gladfelter and J.J. Wymer, eds), Univ. of Chicago Press, p. 163-208.

Thieme H., Dietrich D., URBan B. et VaN KOLFSCHOTEN Th. (1993) - Schöningen (Nordharzvorland). Eine altpaläoli- tische Fundstelle aus dem mittleren Eiszeitalter. Archäologisches Korrespondenzblatt, 23, p. 147-163, 8 fig.

TUFFrEAU A. (1981) - L'Acheuléen dans la France septentrionale. Anthropologie, Brno, XIX/2, p. 171-183, 7 fig.

TUFFREAU A., éd. (1989) - Livret-guide de l'excursion dans la vallée de la Somme. Colloque "L'Acheuléen dans l'Ouest de l'Europe", Saint-Riquier, 115 p., 56 fig.

TUFFrEAu A. (1992) - L'Acheuléen en Europe occidentale d'après les données du bassin de la Somme. I primi abitanti della valle padana : Monte Poggiolo. (C. Peretto ed.), Milano, 1992, p. 41-49, 4 fig.

Tuffreau A. et SOMmé J., dir. (1988) - Le gisement paléolithique moyen de $\mathrm{Bi}$ ache-saint-Vaast (Pas-de-Calais). Vol. I : stratigraphie, environnement, études archéologiques (1 $1^{\text {tre }}$ partie). Mém. Soc. Préhist. Fr., 21, 338 p., illustr.

Tuffreau A., Bouchet J.-P., Moigne A.-M. et Munaut A.-V. (1986) - Les niveaux acheuléens de la moyenne terrasse de la vallée de la Somme à Cagny-l'Épinette. L'Anthr., 90, p. 9-27, 16 fig.

VAN VLIET-LANOE B. (1989) - Observations paléopédologiques sur les gisements de Cagny-la Garenne, Cagny-l'Épinette et Saint-Acheul. Livret-guide de l'excursion dans la vallée de la Somme. Colloque "L'Acheuléen dans l'Ouest de l'Europe", Saint-Riquier, p. 95-99, 1 fig.

VILLA P. (1982) - Conjoinable pieces and site formation processes. American Antiquity, 47, p. 276-90.

VILLA P. (1983) - Terra Amata and the Middle Pleistocene archaeological record of southern France. University of California Publications in Anthropology, 13, $303 \mathrm{p}$.

WERNERT P. (1957) - Stratigraphie, paléontologie et préhistoire des sédiments quaternaires d'Alsace : Achenheim. Mém. Serv. Carte géol. Alsace Lorraine, Strasbourg, 14, 254 p.

Alain TUFFREAU E.R.A. 37 du C.R.A., U.M.R. 9944, C.N.R.S Université des Sciences et Technologies de Lille 59655 Villeneuve-d'Ascq Cedex
Pierre ANTOINE

E.R.A. 37 du C.R.A., U.M.R. 9944, C.N.R.S.

Université des Sciences et Technologies de Lille 59655 Villeneuve-d'Ascq Cedex

Philip G. CHASE University Museum University of Pennsylvania

33rd and Spruce Streets Philadelphia, PA 19104-6398, U.S.A.

Harold L. DIBBLE

Department of Anthropology

University Museum

University of Pennsylvania

33rd and Spruce Streets

Philadelphia, PA 19104-6398, USA

Brooks B. ELLWOOD

Center for Geoenvironmental and Geoarcheological Studies

The University of Texas at Arlington P.O. Box 19049

Arlington, TX 76019, U.S.A.

Thijs van KOLFSCHOTEN

Faculteit der Pre- en Protohistorie

Rijksuniversiteit te Leiden Postbus 9515

2300 R.A. Leiden, Pays-Bas

Agnès LAMOTTE E.R.A. 37 du C.R.A., U.M.R. 9944, C.N.R.S. Université des Sciences et Technologies de Lille 59655 Villeneuve-d'Ascq Cedex

Michel LAURENT Institut de Paléontologie Humaine 1 , rue René-Panhard 75013 Paris

Shannon P. McPHERRON Bishop Museum 1525 Bernice Street P.O. Box 19000A Honnolulu, Hawaii, U.S.A.

Anne-Marie MOIGNE Laboratoire de Préhistoire, U.M.R. 9948, C.N.R.S. Musée de l'Homme 75116 Paris

André V. MUNAUT

Laboratoire de Palynologie et de Dendrochronologie Université catholique de Louvain B-1348 Louvain-la Neuve, Belgique 